\title{
EQUIVALENCE CLASSES OF SUBQUOTIENTS OF PSEUDODIFFERENTIAL OPERATOR MODULES
}

\author{
CHARLES H. CONLEY AND JEANNETTE M. LARSEN
}

\begin{abstract}
Consider the spaces of pseudodifferential operators between tensor density modules over the line as modules of the Lie algebra of vector fields on the line. We compute the equivalence classes of various subquotients of these modules. There is a 2-parameter family of subquotients with any given JordanHölder composition series. In the critical case of subquotients of length 5 , the equivalence classes within each non-resonant 2-parameter family are specified by the intersections of a pencil of conics with a pencil of cubics. In the case of resonant subquotients of length 4 with self-dual composition series, as well as in those of lacunary subquotients of lengths 3 and 4 , equivalence is specified by a single pencil of conics. Non-resonant subquotients of length exceeding 7 admit no non-obvious equivalences. The cases of lengths 6 and 7 are unresolved.
\end{abstract}

\section{INTRODUCTION}

Let $\operatorname{Vect}\left(\mathbb{R}^{m}\right)$ be the Lie algebra of polynomial vector fields on $\mathbb{R}^{m}$. Its natural module $\mathbb{C}\left[x_{1}, \ldots, x_{m}\right]$ has a 1-parameter family of deformations, the tensor density modules $\mathcal{F}_{\lambda}\left(\mathbb{R}^{m}\right)$, the sections of the $\lambda^{\text {th }}$ power of the determinant bundle. These modules are defined for all $\lambda \in \mathbb{C}$, and we write them as

$$
\mathcal{F}_{\lambda}\left(\mathbb{R}^{m}\right):=d x^{\lambda} \mathbb{C}\left[x_{1}, \ldots, x_{m}\right] .
$$

The Lie action $L_{\lambda}$ of $\operatorname{Vect}\left(\mathbb{R}^{m}\right)$ on $\mathcal{F}_{\lambda}\left(\mathbb{R}^{m}\right)$ is

$$
L_{\lambda}(X)\left(d x^{\lambda} f(x)\right):=d x^{\lambda}(X(f)+\lambda f \nabla \cdot X) .
$$

Let $\mathcal{D}_{\lambda, \mu}\left(\mathbb{R}^{m}\right)$ be the space of differential operators from $\mathcal{F}_{\lambda}\left(\mathbb{R}^{m}\right)$ to $\mathcal{F}_{\mu}\left(\mathbb{R}^{m}\right)$, and let $\mathcal{D}_{\lambda, \mu}^{k}\left(\mathbb{R}^{m}\right)$ be the subspace of operators of order $\leq k$. The Lie action $L_{\lambda, \mu}$ of $\operatorname{Vect}\left(\mathbb{R}^{m}\right)$ on $\mathcal{D}_{\lambda, \mu}\left(\mathbb{R}^{m}\right)$ is

$$
L_{\lambda, \mu}(X)(T):=L_{\mu}(X) \circ T-T \circ L_{\lambda}(X) .
$$

It preserves the order filtration, and so one has the subquotient modules

$$
\mathrm{SQ}_{\lambda, \mu}^{k, l}\left(\mathbb{R}^{m}\right):=\mathcal{D}_{\lambda, \mu}^{k}\left(\mathbb{R}^{m}\right) / \mathcal{D}_{\lambda, \mu}^{k-l}\left(\mathbb{R}^{m}\right) .
$$

Note that for $l=1$ these are modules of $k^{\text {th }}$ order symbols. Symbol modules are usually irreducible, so we may think of $l$ as the Jordan-Hölder length of $\mathrm{SQ}_{\lambda, \mu}^{k, l}\left(\mathbb{R}^{m}\right)$. For $l \geq 2$, the subquotient is usually not completely reducible.

The subject of the present article is the following "equivalence question": when are two such subquotients equivalent as modules of $\operatorname{Vect}\left(\mathbb{R}^{m}\right)$ ? This question was first posed by Duval and Ovsienko [DO97], who answered it for modules of the form $\mathcal{D}_{\lambda, \lambda}^{k}\left(\mathbb{R}^{m}\right)$ with $k \leq 2$. In fact they treated smooth differential operators

Received by the editors June 9, 2013 and, in revised form, January 28, 2014.

2010 Mathematics Subject Classification. Primary 17B66.

The first author was partially supported by Simons Foundation Collaboration Grant 207736. 
over arbitrary oriented manifolds, but it is a general phenomenon that the result depends only on the dimension of the manifold, and in the Euclidean case it is the same whether one considers polynomial or smooth functions.

Duval and Ovsienko observed a dichotomy between the 1-dimensional and the multidimensional cases, due to the fact that for $m \geq 2$, the Jordan-Hölder composition series of $\mathrm{SQ}_{\lambda, \mu}^{k, l}\left(\mathbb{R}^{m}\right)$ is determined by the three parameters $\mu-\lambda, k$, and $l$, while for $m=1$ it is determined only by the two parameters $\mu-\lambda-k$ and $l$. This in turn is because the symbol modules of $\mathcal{D}_{\lambda, \mu}\left(\mathbb{R}^{m}\right)$ are tensor density modules for $m=1$, but not for $m \geq 2$.

The work [DO97] inspired several articles. (We note that some authors use a different sign convention, writing $\mathcal{F}_{-\lambda}$ where we write $\mathcal{F}_{\lambda}$.) In the multidimensional case $m \geq 2$, Lecomte, Mathonet, and Tousset [LMT96] determined the equivalence classes of the modules $\mathcal{D}_{\lambda, \lambda}^{k}\left(\mathbb{R}^{m}\right)$ for $k \geq 3$, and Gargoubi and Ovsienko GO96] did the same in the 1-dimensional case. Genuine subquotients were first considered by Lecomte and Ovsienko [LO99], who also made the natural and important generalization to pseudodifferential operators in the 1-dimensional case, allowing the order $k$ to vary continuously. They computed the equivalence classes of the modules $\mathrm{SQ}_{\lambda, \lambda}^{k, l}\left(\mathbb{R}^{m}\right)$ (but only for generic values of $k$ when $m=1$ ).

The equivalence question for arbitrary $(\lambda, \mu)$ was first considered by Mathonet Ma99] and Gargoubi Ga00. In Ma99], all Vect $\left(\mathbb{R}^{m}\right)$-intertwining maps between the modules $\mathcal{D}_{\lambda, \mu}^{k}\left(\mathbb{R}^{m}\right)$ are determined for $m \geq 2$. To our knowledge, the question is not yet settled in the multidimensional case for genuine subquotients $\mathrm{SQ}_{\lambda, \mu}^{k, l}\left(\mathbb{R}^{m}\right)$. The classification of the $\operatorname{Vect}\left(\mathbb{R}^{m}\right)$-maps from differential operator modules $\mathcal{D}_{\lambda, \mu}^{k}\left(\mathbb{R}^{m}\right)$ to tensor density modules $\mathcal{F}_{\nu}\left(\mathbb{R}^{m}\right)$, carried out in Ma00, is closely related.

The equivalences among the modules $\mathcal{D}_{\lambda, \mu}^{k}(\mathbb{R})$ are determined in Ga00. This article contains errors pointed out in [CS04] which call the results at $k=3$ and $\mu-\lambda=1$ or 3 into question, but in fact, as we shall see here, they are correct.

In all of these articles on the equivalence question there is a 1-parameter family of modules with any given composition series. The various answers obtained have a common trait: for small lengths $l$, modules with the same composition series are all equivalent excepting a finite number of special cases, while for larger lengths $l$, modules are equivalent only to their conjugates (adjoints). The most interesting cases involve the critical intermediate lengths, which in these articles are always $l=3$ or $l=4$.

In this article we consider the equivalence classes of the modules $\mathrm{SQ}_{\lambda, \mu}^{k, l}(\mathbb{R})$, the most general 1-dimensional setting. Here there is a 2-parameter family of modules with any given composition series, which causes the critical length to increase to $l=5$. At this length there is a new phenomenon: for each composition series, the equivalence classes are generically six pairs of conjugate modules, determined by the intersections of a certain pencil of conics with a certain pencil of cubics. During the course of the analysis we obtain results in lengths $l \leq 4$ unifying the 1-dimensional results of [DO97, GO96, LO99, Ga00, and Ma00.

We have been unable to resolve the case of length 6 . We can reduce the equivalence question to the computation of a certain Gröbner basis, but the standard software packages were unable to find this basis on the computers available to us. We expect that there are only the obvious equivalences arising from conjugation and the de Rham differential; exceptional equivalences would be interesting. We 
have not resolved the case of length 7 either, but this will be much easier because one has six invariants rather than four. We can prove that in lengths $l \geq 8$ there are no non-obvious equivalences, but we have not included the details here.

We also study "lacunary subquotients", Vect $(\mathbb{R})$-modules whose composition series are missing certain symbol modules. For example, the lacunary subquotients of length 3 composed of the order $k, k-2$, and $k-4$ symbols have the same two parameters for each composition series, and generically they are equivalent if and only if their parameters lie on the same member of the pencil of conics involved in the $l=5$ case. On the other hand, the equivalence classes of the lacunary subquotients of length 4 composed of the order $k, k-2, k-3$, and $k-5$ symbols are determined by a new pencil of conics.

As in GO96, LO99, and Ga00, our main tool is the projective quantization, the decomposition of $\mathcal{D}_{\lambda, \mu}(\mathbb{R})$ under the action of the projective subalgebra of $\operatorname{Vect}(\mathbb{R})$, a copy of $\mathfrak{s l}_{2}$. Generically one has complete reducibility under this subalgebra, in which case it suffices to use the formulas for the action of $\operatorname{Vect}(\mathbb{R})$ with respect to the projective quantization deduced by Cohen-Tretkoff, Manin, and Zagier CMZ97. The exceptions are the resonant cases, where we use the modifications of these formulas obtained in Ga00, and [CS04]. In fact we review these resonant formulas in more detail than is needed for the equivalence question in order to give them in a much simpler form; see Theorem 7.10, One consequence of this simplification is Corollary 7.12, which explains certain initially mysterious factorizations of the non-resonant formulas.

The length 4 resonant case $\mathrm{SQ}_{\lambda, \mu}^{\mu-\lambda+1,4}(\mathbb{R})$ with self-dual composition series is particularly interesting. The special case $\mathcal{D}_{\lambda, \lambda+2}^{3}(\mathbb{R})$ of differential operators was studied in Ga00]: it is the only length $l \leq 4$ composition series for which there are no equivalences other than conjugation. Here we see that in the more general setting of subquotients, equivalence is determined by a single pencil of conics, not one of the pencils of conics arising at $l=5$.

A preliminary outline of these results was given in [Co09, and the non-resonant cases comprise the Ph.D. thesis La12 of the second author. The content of the article is as follows. In Section 2 we state the equivalence question for pseudodifferential operators and recall conjugation, the Adler trace, the de Rham differential, and resonance. In Section 3 we state the complete answer to the equivalence question in all non-resonant cases of length $l \leq 5$, and in Section 4 we do the same in all resonant cases of length $l \leq 4$. In Section 5 we state our results on the lacunary equivalence question, and in Section 6 we discuss the various pencils of conics and cubics which arise. All proofs are given in Section 7, and we conclude in Section 8 with remarks on the equivalence question in higher lengths. Let us mention Proposition 7.15, which states that non-resonant subquotients of length $l \geq 6$ are equivalent if and only if each of their own length 5 subquotients are equivalent, except possibly in certain cases involving the $" \sqrt{19}$ " 1 -cocycles of $\operatorname{Vect}(\mathbb{R})$ discovered by Feigin and Fuchs [FF80.

\section{Definitions And BACKGRound}

Henceforth we work exclusively in one dimension, so we will drop the argument $\mathbb{R}$ and write simply $\mathcal{F}_{\lambda}$ for $d x^{\lambda} \mathbb{C}[x], \mathcal{D}_{\lambda, \mu}$ for the differential operators from $\mathcal{F}_{\lambda}$ to $\mathcal{F}_{\mu}$, and so on. We adopt the standard convention of writing $\delta$ for $\mu-\lambda$ :

$$
\delta(\lambda, \mu):=\mu-\lambda .
$$


We denote the non-negative integers by $\mathbb{N}$ and the positive integers by $\mathbb{Z}^{+}$.

For any $k \in \mathbb{C}$, the $\operatorname{Vect}(\mathbb{R})$-module of pseudodifferential operators from $\mathcal{F}_{\lambda}$ to $\mathcal{F}_{\mu}$ of order in $k-\mathbb{N}$ consists of formal sums:

$$
\Psi_{\lambda, \mu}^{k}:=\left\{d x^{\delta} \sum_{i=0}^{\infty} f_{i}(x) \partial_{x}^{k-i}: f_{i} \in \mathbb{C}[x]\right\} .
$$

The action $L_{\lambda, \mu}$ of $\operatorname{Vect}(\mathbb{R})$ on $\Psi_{\lambda, \mu}^{k}$ is the natural extension of the action on $\mathcal{D}_{\lambda, \mu}$ : $L_{\lambda, \mu}\left(g \partial_{x}\right)\left(d x^{\delta} f \partial_{x}^{r}\right):=d x^{\delta}\left\{\left(g f^{\prime}+(\delta-r) g^{\prime} f\right) \partial_{x}^{r}-f \sum_{s=1}^{\infty}\left(\begin{array}{l}r \\ s\end{array}\right)\left(\lambda+\frac{r-s}{s+1}\right) g^{(s+1)} \partial_{x}^{r-s}\right\}$.

Observe that $\Psi_{\lambda, \mu}^{k-1}$ is a submodule of $\Psi_{\lambda, \mu}^{k}$, and $d x^{\delta} f \partial_{x}^{k} \mapsto d x^{\delta-k} f$ defines a $\operatorname{Vect}(\mathbb{R})$-equivalence from $\Psi_{\lambda, \mu}^{k} / \Psi_{\lambda, \mu}^{k-1}$ to $\mathcal{F}_{\delta-k}$. We extend the definition of $\mathrm{SQ}_{\lambda, \mu}^{k, l}$ to pseudodifferential operators: for $\lambda, \mu$, and $k$ in $\mathbb{C}$ and $l \in \mathbb{Z}^{+}$, set

$$
\mathrm{SQ}_{\lambda, \mu}^{k, l}:=\Psi_{\lambda, \mu}^{k} / \Psi_{\lambda, \mu}^{k-l} .
$$

We shall refer to this $\operatorname{Vect}(\mathbb{R})$-module as a subquotient of length $l$ with composition series $\left\{\mathcal{F}_{\delta-k}, \mathcal{F}_{\delta-k+1}, \ldots, \mathcal{F}_{\delta-k+l-1}\right\}$. This is slightly inaccurate: although $\mathcal{F}_{\nu}$ is irreducible for $\nu \neq 0, \mathcal{F}_{0}$ is a module of length 2 with composition series $\left\{\mathcal{F}_{1}, \mathbb{C}\right\}$. However, the following lemma is clear.

Lemma 2.1. If $\mathrm{SQ}_{\lambda, \mu}^{k, l}$ and $\mathrm{SQ}_{\lambda^{\prime}, \mu^{\prime}}^{k^{\prime}, l^{\prime}}$ are equivalent, then $l=l^{\prime}$ and $\delta-k=\delta^{\prime}-k^{\prime}$.

We may state the equivalence question as follows. Define

$$
n(k, \delta):=\delta-k .
$$

Question. For fixed $n \in \mathbb{C}$ and $l \in \mathbb{Z}^{+}$, what are the $\operatorname{Vect}(\mathbb{R})$-equivalence classes of the set

$$
\left\{\mathrm{SQ}_{\lambda, \mu}^{\delta-n, l}: \lambda, \mu \in \mathbb{C}\right\}
$$

of subquotients of length $l$ with composition series $\left\{\mathcal{F}_{n}, \mathcal{F}_{n+1}, \ldots, \mathcal{F}_{n+l-1}\right\}$ ?

We should note that this question contains the equivalence question for the differential operator modules themselves. Indeed, for $k \in \mathbb{N}$ the length $k+1$ subquotient $\mathrm{SQ}_{\lambda, \mu}^{k, k+1}$ is simply $\mathcal{D}_{\lambda, \mu}^{k}$. More generally, for $l \geq k+2$ we have the canonical $\operatorname{Vect}(\mathbb{R})$-splittings

$$
\Psi_{\lambda, \mu}^{k}=\mathcal{D}_{\lambda, \mu}^{k} \oplus \Psi_{\lambda, \mu}^{-1}, \quad \mathrm{SQ}_{\lambda, \mu}^{k, l}=\mathcal{D}_{\lambda, \mu}^{k} \oplus \mathrm{SQ}_{\lambda, \mu}^{-1, l-k-1} .
$$

Our main result is the answer to the equivalence question in all cases with $l \leq 4$, and in the non-resonant cases with $l=5$. In order to state it efficiently, we recall conjugation, the Adler trace, the de Rham differential, and resonance.

Conjugation of pseudodifferential operators is the adjoint map $T \mapsto T^{*}$ from $\Psi_{\lambda, \mu}^{k}$ to $\Psi_{1-\mu, 1-\lambda}^{k}$ defined by

$$
\left(d x^{\delta} f \partial_{x}^{r}\right)^{*}:=e^{\pi i r} d x^{\delta} \partial_{x}^{r} f=e^{\pi i r} d x^{\delta} \sum_{s=0}^{\infty}\left(\begin{array}{l}
r \\
s
\end{array}\right) f^{(s)} \partial_{x}^{r-s} .
$$

Observe that conjugating twice acts on $\Psi_{\lambda, \mu}^{k}$ as the scalar map $e^{2 \pi i k}$. The following lemma is well-known and easy to prove. 
Lemma 2.2. Conjugation is a Vect $(\mathbb{R})$-equivalence from $\Psi_{\lambda, \mu}^{k}$ to $\Psi_{1-\mu, 1-\lambda}^{k}$. In particular, as $\operatorname{Vect}(\mathbb{R})$-modules,

$$
\mathrm{SQ}_{\lambda, \mu}^{\delta-n, l} \cong \mathrm{SQ}_{1-\mu, 1-\lambda}^{\delta-n, l}
$$

The Adler trace, also known as the non-commutative residue, exists in the category of $\operatorname{Vect}\left(S^{1}\right)$-modules. Algebraically, one passes to this category by simply adjoining $x^{-1}$ to $\operatorname{Vect}(\mathbb{R})$ and all of its modules. The trace is a pairing $\langle\cdot, \cdot\rangle$ between $\Psi_{\lambda, \mu}^{\mathbb{N}+k}\left(S^{1}\right)$ and $\Psi_{\mu, \lambda}^{\mathbb{N}-k}\left(S^{1}\right)$, defined by setting $\left\langle T, T^{\prime}\right\rangle$ to be the residue of the coefficient of $\partial_{x}^{-1}$ in $T \circ T^{\prime}$ when it is written in the form $\sum_{i \in \mathbb{Z}} f_{i} \partial_{x}^{i}$, i.e., the coefficient of $x^{-1}$ in $f_{-1}$. This pairing is known to be non-degenerate, $\operatorname{Vect}\left(S^{1}\right)$-invariant, and symmetric: see for example [CMZ97, LO99, CS04]. It yields the following lemma.

Lemma 2.3. The $\operatorname{Vect}\left(S^{1}\right)$-modules $\mathrm{SQ}_{\lambda, \mu}^{k, l}\left(S^{1}\right)$ and $\mathrm{SQ}_{\mu, \lambda}^{l-2-k, l}\left(S^{1}\right)$ are dual.

Lemmas 2.2 and 2.3 both originate in the fact that the $\operatorname{Vect}\left(S^{1}\right)$-modules $\mathcal{F}_{\nu}\left(S^{1}\right)$ and $\mathcal{F}_{1-\nu}\left(S^{1}\right)$ are dual. The consequence of Lemma 2.3 relevant here is the following corollary. In the category of $\operatorname{Vect}\left(S^{1}\right)$-modules it follows from Lemma 4.2 in [Co05. To prove it in the category of $\operatorname{Vect}(\mathbb{R})$-modules, observe that $\mathrm{SQ}_{\lambda, \mu}^{k, l}$ is precisely the subspace of $\mathrm{SQ}_{\lambda, \mu}^{k, l}\left(S^{1}\right)$ on which the constant vector field $\partial_{x}$ acts locally nilpotently. Therefore any $\partial_{x}$-equivalence of $S^{1}$-subquotients must restrict to a $\partial_{x}$-equivalence of the $\mathbb{R}$-subquotients they contain.

Corollary 2.4. As Vect $(\mathbb{R})$-modules,

$$
\mathrm{SQ}_{\lambda, \mu}^{\mu-\lambda-n, l} \cong \mathrm{SQ}_{\lambda^{\prime}, \mu^{\prime}}^{\mu^{\prime}-\lambda^{\prime}-n, l} \text { if and only if } \mathrm{SQ}_{\mu, \lambda}^{l-2+n+\lambda-\mu, l} \cong \mathrm{SQ}_{\mu^{\prime}, \lambda^{\prime}}^{l-2+n+\lambda^{\prime}-\mu^{\prime}, l} \text {. }
$$

The following definition will permit us to take advantage of these symmetries.

Definition. Let $\gamma(\lambda, \mu):=3(\lambda+\mu-1)^{2}$ and $N_{l}(n):=n+\frac{1}{2} l-1$.

For fixed $l$ and $n$, Lemma 2.2 implies that the equivalence class of $\mathrm{SQ}_{\lambda, \mu}^{\delta-n, l}$ depends only on $\gamma$ and $\delta$. By Corollary 2.4, the equations defining this equivalence class are symmetric under $\left(N_{l}, \gamma, \delta\right) \mapsto\left(-N_{l}, \gamma,-\delta\right)$. Therefore we will give the equations in terms of these coordinates.

Keep in mind that $(\gamma, \delta)$ specifies a conjugate pair of values of $(\lambda, \mu)$ rather than a single value. In fact, many of our formulas involve $\gamma^{1 / 2}$. Although the statements of our main results are independent of the choice of sign of the square root, for concreteness we specify

$$
\gamma^{1 / 2}(\lambda, \mu):=\sqrt{3}(\lambda+\mu-1) .
$$

Henceforth we will always use the notation

$$
\left(\gamma^{\prime}, \delta^{\prime}\right):=\left(\gamma\left(\lambda^{\prime}, \mu^{\prime}\right), \delta\left(\lambda^{\prime}, \mu^{\prime}\right)\right) .
$$

We make the following definition in order to be able to regard the equivalence class of $\mathrm{SQ}_{\lambda, \mu}^{\delta-n, l}$ as a subset of the $(\gamma, \delta)$-plane.

Definition. $\operatorname{EC}_{n}^{l}(\gamma, \delta):=\left\{\left(\gamma^{\prime}, \delta^{\prime}\right) \in \mathbb{C}^{2}: \operatorname{SQ}_{\lambda^{\prime}, \mu^{\prime}}^{\delta^{\prime}-n} \cong \mathrm{SQ}_{\lambda, \mu}^{\delta-n, l}\right\}$

The de Rham differential is

$$
d:=d x \partial_{x}: \mathcal{F}_{0} \rightarrow \mathcal{F}_{1},
$$


the only non-scalar Vect $(\mathbb{R})$-map between tensor density modules. It gives rise to an equivalence between subquotients of arbitrary length, which we now describe. Write $L_{d}$ and $R_{d}$ for left and right composition with $d$, respectively:

$$
\begin{array}{ll}
L_{d}: \Psi_{\lambda, 0}^{k} \rightarrow \Psi_{\lambda, 1}^{k+1}, & T \mapsto d \circ T, \\
R_{d}: \Psi_{1, \mu}^{k} \rightarrow \Psi_{0, \mu}^{k+1}, & T \mapsto T \circ d .
\end{array}
$$

These maps are both $\operatorname{Vect}(\mathbb{R})$-isomorphisms, which induce Vect $(\mathbb{R})$-isomorphisms

$$
L_{d}: \mathrm{SQ}_{\lambda, 0}^{-\lambda-n, l} \rightarrow \mathrm{SQ}_{\lambda, 1}^{1-\lambda-n, l}, \quad R_{d}: \mathrm{SQ}_{1, \mu}^{\mu-1-n, l} \rightarrow \mathrm{SQ}_{0, \mu}^{\mu-n, l} .
$$

Observe that the two cases form a conjugate pair, so in $(\gamma, \delta)$-coordinates they appear as a single case. Thus we have:

Lemma 2.5. For all $l, n$, and $\nu, \operatorname{EC}_{n}^{l}\left(3(\nu+1)^{2}, \nu\right)=\operatorname{EC}_{n}^{l}\left(3 \nu^{2}, \nu+1\right)$.

The interplay between (1) and the maps $L_{d}$ and $R_{d}$ gives:

Lemma 2.6. For all $\lambda$ and $\mu$, we have the $\operatorname{Vect}(\mathbb{R})$-splittings

$$
\begin{array}{llrl}
\mathcal{D}_{\lambda, 1}=\mathcal{D}_{\lambda, 1}^{0} \oplus L_{d}\left(\mathcal{D}_{\lambda, 0}\right), & \Psi_{\lambda, 0}^{-1}=\Psi_{\lambda, 0}^{-2} \oplus L_{d}^{-1}\left(\mathcal{D}_{\lambda, 1}^{0}\right), \\
\mathcal{D}_{0, \mu}=\mathcal{D}_{0, \mu}^{0} \oplus R_{d}\left(\mathcal{D}_{1, \mu}\right), & \Psi_{1, \mu}^{-1}=\Psi_{1, \mu}^{-2} \oplus R_{d}^{-1}\left(\mathcal{D}_{0, \mu}^{0}\right), \\
\mathcal{D}_{0,1}=\mathcal{D}_{0,1}^{1} \oplus L_{d} R_{d}\left(\mathcal{D}_{1,0}\right), & \Psi_{1,0}^{-1}=\Psi_{1,0}^{-3} \oplus L_{d}^{-1} R_{d}^{-1}\left(\mathcal{D}_{0,1}^{1}\right) .
\end{array}
$$

Resonance is the failure of complete reducibility under the action of the projective subalgebra of $\operatorname{Vect}(\mathbb{R})$. This subalgebra is

$$
\mathfrak{a}:=\operatorname{Span}_{\mathbb{C}}\left\{\partial_{x}, x \partial_{x}, x^{2} \partial_{x}\right\},
$$

an isomorphic copy of $\mathfrak{s l}_{2}$. Its Casimir operator, the central element

$$
\Omega:=\left(x \partial_{x}\right) \otimes\left(x \partial_{x}\right)-\left(x \partial_{x}\right)-\left(x^{2} \partial_{x}\right) \otimes\left(\partial_{x}\right)
$$

of its universal enveloping algebra, acts on $\mathcal{F}_{\nu}$ by the scalar $L_{\nu}(\Omega)=\nu^{2}-\nu$.

The module $\mathrm{SQ}_{\lambda, \mu}^{\delta-n, l}$ cannot be resonant unless its composition series has repeated Casimir eigenvalues. Since the values of $\nu^{2}-\nu$ are symmetric around $\nu=\frac{1}{2}$, this occurs if and only if $(n+i)+(n+j)$ is 1 for some $0 \leq i<j \leq l-1$, i.e., $l \geq 2$ and $-2 n \in\{0,1, \ldots, 2 l-4\}$. In fact, $\mathrm{SQ}_{\lambda, \mu}^{\delta-n, l}$ is generically resonant for such values of $n$, so we make the following definition.

Definition. $\mathrm{SQ}_{\lambda, \mu}^{\delta-n, l}$ is resonant if $n \in\left\{0,-\frac{1}{2},-1,-\frac{3}{2}, \ldots, 2-l\right\}$. We say that it is integral resonant or half-integral resonant depending on whether $n$ is integral or half-integral.

Note that the set of resonant values of $N_{l}$ is $\left\{0, \pm \frac{1}{2}, \pm 1, \pm \frac{3}{2}, \ldots, \pm\left(\frac{1}{2} l-1\right)\right\}$. Its symmetry around zero is a consequence of Lemma 2.3. Resonant pseudodifferential operator modules were studied in detail in Ga00] and [CS04.

\section{NON-RESONANT RESULtS}

In this section we answer the equivalence question in all non-resonant cases of length $l \leq 5$. Proofs will be deferred to Section 7. We present the results in order of increasing length. Note that any subquotient $\mathrm{SQ}_{\lambda, \mu}^{\delta-n, l}$ of length $l$ contains two "subsubquotients" of lengths $l-1$, namely, $\mathrm{SQ}_{\lambda, \mu}^{\delta-n, l-1}$ and $\mathrm{SQ}_{\lambda, \mu}^{\delta-n-1, l-1}$. It is simple to check that a necessary condition for the equivalence of two length $l$ subquotients is the pairwise equivalence of their sub-subquotients, but as can be seen from our 
results, this condition is in general not sufficient. However, we will see in Section 8 that it is sufficient in lengths $l \geq 6$, with the possible exception of certain special length 7 cases.

Keep in mind that by Lemma 2.1 $l$ and $n$ are invariant under equivalence: they are complete invariants for the composition series of $\mathrm{SQ}_{\lambda, \mu}^{\delta-n, l}$. For $l=1$ there is nothing to prove: there are no resonant cases and $\mathrm{SQ}_{\lambda, \mu}^{\delta-n, 1}$ is always equivalent to $\mathcal{F}_{n}$. For $l=2$, the only resonant case is $n=0$. For $n \neq 0$ it is well-known that there is only one equivalence class: for all $(\lambda, \mu)$,

$$
\mathrm{SQ}_{\lambda, \mu}^{\delta-n, 2} \cong \mathcal{F}_{n} \oplus \mathcal{F}_{n+1}
$$

See for example Lemma 7.9 of [LO99], Lemma 3.3 of [CS04, or page 72 of [Co09].

In order to state the results for $3 \leq l \leq 5$, we give slight modifications of (6), (7), and (8) of [Co05] and make a convenient definition:

$$
\begin{aligned}
B_{m+2, m}(\gamma, \delta):= & \gamma-\left[(2 m+1) \delta+\left(m^{2}+m+1\right)\right], \\
B_{m+3, m}(\gamma, \delta):= & \gamma^{3 / 2}-3 \gamma^{1 / 2}[(m+1) \delta+1], \\
B_{m+4, m}(\gamma, \delta):= & \gamma^{2}-\gamma\left[2(2 m+3) \delta-\left(2 m^{2}+6 m-3\right)\right] \\
& -\frac{3}{5} m(m+3)\left[2(2 m+3) \delta+\left(m^{2}+3 m+6\right)\right] .
\end{aligned}
$$

Definition. Two subquotients $\mathrm{SQ}_{\lambda, \mu}^{\delta-n, l}$ and $\mathrm{SQ}_{\lambda^{\prime}, \mu^{\prime}}^{\delta^{\prime}}$ are said to induce simultaneous vanishing of the functions $f_{1}(\gamma, \delta), \ldots, f_{r}(\gamma, \delta)$ if for all $s, f_{s}(\gamma, \delta)$ and $f_{s}\left(\gamma^{\prime}, \delta^{\prime}\right)$ are either both zero or both non-zero.

Henceforth we will use the Pochhammer symbol $(x)_{r}$ for the falling factorial:

$$
(x)_{r}:=x(x-1) \cdots(x-r+1), \quad(x)_{0}:=1 .
$$

3.1. Length $l=3$. Here the set of resonant values of $n$ is $\left\{-1,-\frac{1}{2}, 0\right\}$, and so that of $N_{3}=n+\frac{1}{2}$ is $\left\{0, \pm \frac{1}{2}\right\}$. We will need the formula for $B_{n+2, n}$ in terms of $N_{3}$ :

$$
B_{n+2, n}=\gamma-\left[2 N_{3} \delta+N_{3}^{2}+\frac{3}{4}\right] .
$$

Proposition 3.1. For $n$ non-resonant, $\mathrm{SQ}_{\lambda, \mu}^{\delta-n, 3}$ and $\mathrm{SQ}_{\lambda^{\prime}, \mu^{\prime}}^{\delta^{\prime}-n, 3}$ are equivalent if and only if they induce simultaneous vanishing of

$$
\left(\delta-N_{3}+\frac{1}{2}\right)_{2} B_{n+2, n} .
$$

The equivalence class where (3) vanishes splits as $\mathcal{F}_{n} \oplus \mathcal{F}_{n+1} \oplus \mathcal{F}_{n+2}$.

Let us make some remarks on this proposition and explain how to use it to recover earlier length 3 results. First, $\left(\delta-N_{3}+\frac{1}{2}\right)_{2}=0$ if and only if the order $k$ is 0 or 1 . In these cases the equivalence class is split because of (11).

Observe that (3) has the symmetry promised by Corollary 2.4. To verify Lemma 2.5 directly, check that the two $(\gamma, \delta)$-values $\left(3(\nu+1)^{2}, \nu\right)$ and $\left(3 \nu^{2}, \nu+1\right)$ give the same value in (3), namely, $\left(\nu-N_{3}+\frac{3}{2}\right)_{3}\left(3 \nu+N_{3}+\frac{3}{2}\right)$.

DO97] gives the equivalence classes of the modules $\mathcal{D}_{\lambda, \lambda}^{2}=\mathrm{SQ}_{\lambda, \lambda}^{2,3}$ : the $\lambda$-values 0 and 1 form the split class, and all other $\lambda$-values form the other class. To recover this result, note that $B_{0,-2}(\gamma, 0)=12 \lambda(\lambda-1)$.

LO99] gives the equivalence classes of the modules $\mathrm{SQ}_{\lambda, \lambda}^{k, 3}$ with $k \notin \frac{1}{2} \mathbb{N}$ : the two $\lambda$-roots of $B_{2-k,-k}(\gamma, 0)=12 \lambda(\lambda-1)-(k-2)(k+1)$ form the split class, and all other $\lambda$-values form the other class. 
Ga00] gives the equivalence classes of the modules $\mathcal{D}_{\lambda, \mu}^{2}=\mathrm{SQ}_{\lambda, \mu}^{2,3}$ : modules with a given $\delta$ have two classes, the conjugate pair $\left\{\mathcal{D}_{0, \delta}^{2}, \mathcal{D}_{1-\delta, 1}^{2}\right\}$ and all the others. This result is a manifestation of Lemma 2.6. To recover it in the non-resonant cases, note that $B_{\delta, \delta-2}(\gamma, \delta)=12 \lambda(\mu-1)$.

Ma00] gives all projections $\mathcal{D}_{\lambda, \mu}^{k} \rightarrow \mathcal{F}_{\mu-\lambda-k+2}$. In the non-resonant case they exist if and only if $\mathrm{SQ}_{\lambda, \mu}^{k, 3}$ is in the split equivalence class, i.e., $B_{n+2, n}(\gamma, \delta)=0$ (and $k \in 2+\mathbb{N}$ ). We mention that the hyperbola in Definition 3.3 of [Ma00] is

$$
3 B_{n+2, n}(\gamma, \delta)=4(3 \lambda+k-2)(3 \mu-k-1)+(k-2)(k+1)=0 .
$$

3.2. Length $l=4$. Here the set of resonant values of $n$ is $\left\{-2,-\frac{3}{2},-1,-\frac{1}{2}, 0\right\}$, and so that of $N_{4}=n+1$ is $\left\{0, \pm \frac{1}{2}, \pm 1\right\}$. In terms of $N_{4}$,

$$
\begin{aligned}
B_{n+2, n} & =\gamma-\left[\left(2 N_{4}-1\right) \delta+\left(N_{4}^{2}-N_{4}+1\right)\right], \\
B_{n+3, n+1} & =\gamma-\left[\left(2 N_{4}+1\right) \delta+\left(N_{4}^{2}+N_{4}+1\right)\right], \\
B_{n+3, n} & =\gamma^{3 / 2}-3 \gamma^{1 / 2}\left[N_{4} \delta+1\right] .
\end{aligned}
$$

Proposition 3.2. For $n$ non-resonant, $\mathrm{SQ}_{\lambda, \mu}^{\delta-n, 4}$ and $\mathrm{SQ}_{\lambda^{\prime}, \mu^{\prime}}^{\delta^{\prime}-n, 4}$ are equivalent if and only if they induce simultaneous vanishing of

$$
\left(\delta-N_{4}+1\right)_{2} B_{n+2, n}, \quad\left(\delta-N_{4}\right)_{2} B_{n+3, n+1}, \quad\left(\delta-N_{4}+1\right)_{3} B_{n+3, n} .
$$

Note that all eight possible sets of vanishings occur, so there are eight equivalence classes. However, all three polynomials vanish only when there is vanishing among the Pochhammer symbols. This occurs when the order $\delta-n$ is 0,1 , or 2 , the situation of (11). For example, all modules with $\delta=N_{4}$ are in this equivalence class. We now use Proposition 3.2 to recover earlier length 4 results.

G096] computes the equivalence classes of $\mathcal{D}_{\lambda, \lambda}^{3}=\mathrm{SQ}_{\lambda, \lambda}^{3,4}$ : there are four, given by the sets of $\lambda$-values

$$
\left\{\frac{1}{2}\right\}, \quad\{0,1\}, \quad\left\{\frac{1}{6}(3 \pm \sqrt{21})\right\}, \quad \mathbb{C} \backslash\left\{0, \frac{1}{2}, 1, \frac{1}{6}(3 \pm \sqrt{21})\right\} .
$$

To prove this, apply the proposition to the following proportionalities at $(\gamma, 0)$ :

$$
B_{-1,-3} \propto 3 \lambda^{2}-3 \lambda-1, \quad B_{-2,0} \propto \lambda(\lambda-1), \quad B_{0,-3} \propto \lambda\left(\lambda-\frac{1}{2}\right)(\lambda-1) .
$$

LO99] gives the generic equivalence class of the modules $\mathrm{SQ}_{\lambda, \lambda}^{k, 4}$ with $k \notin \frac{1}{2} \mathbb{N}$ : all those on which none of the functions $t_{k}^{2}, t_{k-1}^{2}$, and $t_{k}^{3}$ defined in Proposition 7.10 of that article vanish are equivalent. To prove this, check the proportionalities

$$
t_{k}^{2} \propto B_{2-k,-k}(\gamma, 0), \quad t_{k-1}^{2} \propto B_{3-k, 1-k}(\gamma, 0), \quad t_{k}^{3} \propto B_{3-k,-k}(\gamma, 0) .
$$

Ga00] gives the equivalence classes of the modules $\mathcal{D}_{\lambda, \mu}^{3}=\mathrm{SQ}_{\lambda, \mu}^{3,4}$, superseding G096]. To reconstruct the results in the non-resonant cases, note that here $n=$ $\delta-3$ and the Pochhammer symbols in Proposition 3.2 never vanish, so the equivalence classes are determined by the vanishing of

$B_{\delta-1, \delta-3} \propto(3 \lambda+1)(3 \mu-4)+1, B_{\delta, \delta-2} \propto \lambda(\mu-1), B_{\delta, \delta-3} \propto \lambda(\mu-1)(\lambda+\mu-1)$.

Ma00] gives all projections $\mathcal{D}_{\lambda, \mu}^{k} \rightarrow \mathcal{F}_{\mu-\lambda-k+3}$. In the non-resonant case, they exist if and only if both $B_{n+3, n+1}$ (see (44)) and $B_{n+3, n} \propto \lambda(\mu-1)(\lambda+\mu-1)$ are 
zero. Thus for $k=3$ they exist if and only if either $\lambda=0$ or $\mu=1$, and for $k \in 4+\mathbb{N}$ they exist only in the two self-conjugate cases

$$
\lambda=-\frac{1}{3}(k-3) \pm \frac{1}{6} \sqrt{k(k-3)}, \quad \mu=1-\lambda .
$$

3.3. Length $l=5$. We have seen that in length $l \leq 4$, almost all subquotients $\mathrm{SQ}_{\lambda, \mu}^{\delta-n, l}$ with a given $n$ are equivalent. At $l=5$ there is a new phenomenon: equivalence is determined by two rational invariants. We begin with a special case of the definition of simultaneous vanishing:

Definition. Two non-resonant subquotients $\mathrm{SQ}_{\lambda, \mu}^{\delta-n, l}$ and $\mathrm{SQ}_{\lambda^{\prime}, \mu^{\prime}}^{\delta^{\prime}-n, l}$ are said to satisfy the simultaneous vanishing condition (SVC) if for all $(i, j)$ such that $0 \leq j \leq$ $i-2$ and $2 \leq i \leq l-1$, they induce simultaneous vanishing of

$$
(\delta-n-j)_{i-j} B_{n+i, n+j}(\gamma, \delta) .
$$

As before, the Pochhammer symbols vanish only in the situation of (1). With this definition we can restate Propositions 3.1 and 3.2 concisely:

Proposition 3.3. For $l \leq 4$, non-resonant subquotients $\mathrm{SQ}_{\lambda, \mu}^{\delta-n, l}$ and $\mathrm{SQ}_{\lambda^{\prime}, \mu^{\prime}}^{\delta^{\prime}-n, l}$ are equivalent if and only if they satisfy the SVC.

This is not true for $l \geq 5$. We expect that in length $l \geq 6$, the conjugation and de Rham equivalences described in Lemmas 2.2 and 2.5 are the only equivalences. However, in length 5 there are more. Here the set of resonant values of $n$ is $\left\{-3,-\frac{5}{2},-2,-\frac{3}{2},-1,-\frac{1}{2}, 0\right\}$, and so that of $N_{5}=n+\frac{3}{2}$ is $\left\{0, \pm \frac{1}{2}, \pm 1, \pm \frac{3}{2}\right\}$. In terms of $N_{5}$,

$$
\begin{aligned}
B_{n+2, n} & =\gamma-\left[2\left(N_{5}-1\right) \delta+\left(N_{5}-1\right)^{2}+\frac{3}{4}\right] \\
B_{n+3, n+1} & =\gamma-\left[2 N_{5} \delta+N_{5}^{2}+\frac{3}{4}\right] \\
B_{n+4, n+2} & =\gamma-\left[2\left(N_{5}+1\right) \delta+\left(N_{5}+1\right)^{2}+\frac{3}{4}\right] \\
B_{n+3, n} & =\gamma^{3 / 2}-3 \gamma^{1 / 2}\left[\left(N_{5}-\frac{1}{2}\right) \delta+1\right] \\
B_{n+4, n+1} & =\gamma^{3 / 2}-3 \gamma^{1 / 2}\left[\left(N_{5}+\frac{1}{2}\right) \delta+1\right], \\
B_{n+4, n} & =\gamma^{2}-2 \gamma\left[2 N_{5} \delta-N_{5}^{2}+\frac{15}{4}\right]-\frac{3}{5}\left(N_{5}^{2}-\frac{9}{4}\right)\left[4 N_{5} \delta+N_{5}^{2}+\frac{15}{4}\right] .
\end{aligned}
$$

The invariants which will determine equivalence are

$$
\begin{aligned}
I_{n}(\gamma, \delta) & :=B_{n+4, n} / B_{n+4, n+2} B_{n+2, n}, \\
J_{n}(\gamma, \delta) & :=B_{n+4, n} B_{n+3, n+1} / B_{n+4, n+1} B_{n+3, n}, \\
K_{n}(\gamma, \delta) & :=B_{n+4, n+2} B_{n+3, n+1} B_{n+2, n} / B_{n+4, n+1} B_{n+3, n} .
\end{aligned}
$$

Note that $K_{n}=J_{n} / I_{n}$. We now state our main result in the non-resonant case.

Theorem 3.4. For $n$ non-resonant, the subquotients $\mathrm{SQ}_{\lambda, \mu}^{\delta-n, 5}$ and $\mathrm{SQ}_{\lambda^{\prime}, \mu^{\prime}}^{\delta^{\prime}-n}$ are equivalent if and only if they satisfy the $S V C$, and either $(\delta-n)_{4}\left(\delta^{\prime}-n\right)_{4}=0$ or $(\delta-n)_{4}\left(\delta^{\prime}-n\right)_{4} \neq 0$, and one of the following mutually exclusive conditions holds:

(i) At least two of $B_{n+4, n}, B_{n+4, n+2} B_{n+2, n}$, and $B_{n+4, n+1} B_{n+3, n} B_{n+3, n+1}$ are zero.

(ii) $B_{n+4, n} B_{n+4, n+2} B_{n+2, n}$ is not zero, $B_{n+4, n+1} B_{n+3, n} B_{n+3, n+1}$ is zero, and

$$
I_{n}(\gamma, \delta)=I_{n}\left(\gamma^{\prime}, \delta^{\prime}\right)
$$


(iii) $B_{n+4, n} B_{n+4, n+1} B_{n+3, n} B_{n+3, n+1}$ is not zero, $B_{n+4, n+2} B_{n+2, n}$ is zero, and

$$
J_{n}(\gamma, \delta)=J_{n}\left(\gamma^{\prime}, \delta^{\prime}\right) .
$$

(iv) $B_{n+4, n+1} B_{n+3, n} B_{n+4, n+2} B_{n+3, n+1} B_{n+2, n}$ is not zero, $B_{n+4, n}$ is zero, and

$$
K_{n}(\gamma, \delta)=K_{n}\left(\gamma^{\prime}, \delta^{\prime}\right)
$$

(v) $B_{n+4, n} B_{n+4, n+1} B_{n+3, n} B_{n+4, n+2} B_{n+3, n+1} B_{n+2, n}$ is not zero, and

$$
I_{n}(\gamma, \delta)=I_{n}\left(\gamma^{\prime}, \delta^{\prime}\right), \quad J_{n}(\gamma, \delta)=J_{n}\left(\gamma^{\prime}, \delta^{\prime}\right)
$$

Let us give a preliminary interpretation of this theorem. Recall that $n$ and thus also $N_{5}$ may be regarded as fixed because they determine the composition series of $\mathrm{SQ}_{\lambda, \mu}^{\delta-n, 5}$ and so are invariant under equivalence. By Theorem 3.4 (v), away from the zero loci of the six $B_{n+i, n+j}$ 's (6) in the $(\gamma, \delta)$-plane, $I_{n}$ and $J_{n}$ are complete invariants for the equivalence classes of the subquotients $\mathrm{SQ}_{\lambda, \mu}^{\delta-n, 5}$.

It is not difficult to see that the level curves of $I_{n}$ form the pencil of conics passing through four fixed points depending only on $N_{5}$, and the level curves of $J_{n}$ form the pencil of cubics passing through nine fixed points depending only on $N_{5}$ (one of them is on the line at infinity). Thus generically, $\mathrm{SQ}_{\lambda, \mu}^{\delta-n, 5} \cong \mathrm{SQ}_{\lambda^{\prime}, \mu^{\prime}}^{\delta^{\prime}-n}$ if and only if $(\gamma, \delta)$ and $\left(\gamma^{\prime}, \delta^{\prime}\right)$ lie on the same conic and the same cubic in these pencils. Put differently, $\operatorname{EC}_{n}^{5}(\gamma, \delta)$ is the intersection of the conic and the cubic through $(\gamma, \delta)$. This intersection is usually six points in $(\gamma, \delta)$-space, so the equivalence classes are usually six pairs of conjugate subquotients.

We shall describe the pencils of conics and cubics in detail in Section 6. In particular, we shall recover the results of [LO99] and Ga00 in length 5: for either $\delta$ fixed at 0 or the order $k=\delta-n$ fixed at 4 , generically the only non-trivial equivalence is conjugation.

\section{Resonant Results}

In this section we answer the equivalence question in all resonant cases of length $l \leq 4$, and in the self-dual resonant case of length 5 . As before, proofs are deferred to Section 7 For the resonant modules $\mathcal{D}_{\lambda, \mu}^{k}$, the results match those of [Ga00].

We recall relevant material from Section 2] $\mathrm{SQ}_{\lambda, \mu}^{\delta-n, l}$ is resonant if its composition series $\left\{\mathcal{F}_{n}, \mathcal{F}_{n+1}, \ldots, \mathcal{F}_{n+l-1}\right\}$ contains a pair of tensor density modules of degrees symmetric around $\frac{1}{2}$. The duality described by Lemma 2.3 pairs $n$ with $2-l-n$, and hence $N_{l}:=n+\frac{1}{2} l-1$ with $-N_{l}$. Thus resonance occurs for

$$
n \in\left\{0,-\frac{1}{2},-1,-\frac{3}{2}, \ldots, 1-\frac{1}{2} l\right\}, \quad \text { i.e., } \quad N_{l} \in\left\{0, \pm \frac{1}{2}, \pm 1, \pm \frac{3}{2}, \ldots, \pm\left(\frac{1}{2} l-1\right)\right\} .
$$

In particular, for $l=1$ there are no resonant cases, for $l=2$ the only resonant $n$-value is 0 , for $l=3$ the resonant $n$-values are $-1,-\frac{1}{2}$, and 0 , and for $l=4$ they are $-2,-\frac{3}{2},-1,-\frac{1}{2}$, and 0 .

We begin by extending the definition of the SVC to the resonant cases and stating a proposition which resolves the majority of resonant cases of length $l \leq 4$. We will see that the self-dual cases, where $n=1-\frac{1}{2} l$ and $N_{l}=0$, tend to be exceptional.

Definition. For $n$ half-integral resonant, the simultaneous vanishing condition is the same as in the non-resonant case. For $n$ integral resonant, two subquotients $\mathrm{SQ}_{\lambda, \mu}^{\delta-n, l}$ and $\mathrm{SQ}_{\lambda^{\prime}, \mu^{\prime}}^{\delta^{\prime}-n}$ are said to satisfy the simultaneous vanishing condition if they satisfy the non-resonant SVC and in addition induce simultaneous vanishing of $\delta \gamma^{1 / 2}$. 
Proposition 4.1. For $l \leq 3$ or $l=4$ and $n=-\frac{1}{2}$ or $-\frac{3}{2}$, resonant subquotients $\mathrm{SQ}_{\lambda, \mu}^{\delta-n, l}$ and $\mathrm{SQ}_{\lambda^{\prime}, \mu^{\prime}}^{\delta^{\prime}-n, l}$ are equivalent if and only if they satisfy the SVC.

Let us write the SVC explicitly in each of these cases. In the self-dual case $l=2$ and $n=0$, we have the subquotients $\operatorname{SQ}_{\lambda, \mu}^{\delta, 2}$ with composition series $\left\{\mathcal{F}_{0}, \mathcal{F}_{1}\right\}$. By the proposition, two such are equivalent if and only if they induce simultaneous vanishing of $\delta \gamma^{1 / 2}$. The equivalence class where $\delta \gamma^{1 / 2}$ vanishes splits as $\mathcal{F}_{0} \oplus \mathcal{F}_{1}$ : the factor $\gamma^{1 / 2}$ reflects the splitting of self-conjugate modules into symmetric and skew-symmetric parts, and the factor $\delta$ reflects (1).

For reference, we write out those functions $B_{n+i, n}(\gamma, \delta)$ which arise in lengths 3 and 4 , arranged in dual pairs. Those with $i=2$ are

$$
\begin{aligned}
B_{\frac{3}{2},-\frac{1}{2}}=\gamma-\frac{3}{4} & \\
B_{2,0}=\gamma-\delta-1, & B_{1,-1}=\gamma+\delta-1, \\
B_{\frac{5}{2}, \frac{1}{2}}=\gamma-2 \delta-\frac{7}{4}, & B_{\frac{1}{2},-\frac{3}{2}}=\gamma+2 \delta-\frac{7}{4}, \\
B_{3,1}=\gamma-3 \delta-3, & B_{0,-2}=\gamma+3 \delta-3,
\end{aligned}
$$

and those with $i=3$ are

$$
\begin{aligned}
& B_{2,-1}=\gamma^{1 / 2}(\gamma-3) \text {, } \\
& B_{\frac{5}{2},-\frac{1}{2}}=\gamma^{1 / 2}\left(\gamma-\frac{3}{2} \delta-3\right), \quad B_{\frac{3}{2},-\frac{3}{2}}=\gamma^{1 / 2}\left(\gamma+\frac{3}{2} \delta-3\right) \text {, } \\
& B_{3,0}=\gamma^{1 / 2}(\gamma-3 \delta-3), \quad B_{1,-2}=\gamma^{1 / 2}(\gamma+3 \delta-3) .
\end{aligned}
$$

In the self-dual length 3 case $n=-\frac{1}{2}, \mathrm{SQ}_{\lambda, \mu}^{\delta+\frac{1}{2}, 3}$ and $\mathrm{SQ}_{\lambda^{\prime}, \mu^{\prime}}^{\delta^{\prime}+\frac{1}{2}, 3}$ are equivalent if and only if they induce simultaneous vanishing of

$$
\left(\delta+\frac{1}{2}\right)_{2} B_{\frac{3}{2},-\frac{1}{2}} \cdot
$$

The equivalence class where this quantity vanishes splits as $\mathcal{F}_{-1 / 2} \oplus \mathcal{F}_{1 / 2} \oplus \mathcal{F}_{3 / 2}$. In fact, $\gamma=\frac{3}{4}$ reduces to $\lambda+\mu=\frac{1}{2}$ or $\frac{3}{2}$, the situation of Proposition 9.1(b) in Ga00] (see also Section 8.2 of [CS04]).

In the length 3 case $n=0, \mathrm{SQ}_{\lambda, \mu}^{\delta, 3}$ and $\mathrm{SQ}_{\lambda^{\prime}, \mu^{\prime}}^{\delta^{\prime}, 3}$ are equivalent if and only if they induce simultaneous vanishing of

$$
\delta \gamma^{1 / 2}, \quad(\delta)_{2} B_{2,0} .
$$

In the dual case $l=3$ and $n=-1, \mathrm{SQ}_{\lambda, \mu}^{\delta+1,3}$ and $\mathrm{SQ}_{\lambda^{\prime}, \mu^{\prime}}^{\delta^{\prime}+1,3}$ are equivalent if and only if they induce simultaneous vanishing of

$$
\delta \gamma^{1 / 2}, \quad(\delta+1)_{2} B_{1,-1} .
$$

In the length 4 resonant cases with $n=-\frac{1}{2}, \mathrm{SQ}_{\lambda, \mu}^{\delta+\frac{1}{2}, 4}$ and $\mathrm{SQ}_{\lambda^{\prime}, \mu^{\prime}}^{\delta^{\prime}+\frac{1}{2}, 4}$ are equivalent if and only if they induce simultaneous vanishing of

$$
\left(\delta+\frac{1}{2}\right)_{2} B_{\frac{3}{2},-\frac{1}{2}}, \quad\left(\delta-\frac{1}{2}\right)_{2} B_{\frac{5}{2}, \frac{1}{2}}, \quad\left(\delta+\frac{1}{2}\right)_{3} B_{\frac{5}{2},-\frac{1}{2}} .
$$

In the dual case $l=4$ and $n=-\frac{3}{2}, \mathrm{SQ}_{\lambda, \mu}^{\delta+\frac{3}{2}, 4}$ and $\mathrm{SQ}_{\lambda^{\prime}, \mu^{\prime}}^{\delta^{\prime}+\frac{3}{2}, 4}$ are equivalent if and only if they induce simultaneous vanishing of

$$
\left(\delta+\frac{1}{2}\right)_{2} B_{\frac{3}{2},-\frac{1}{2}}, \quad\left(\delta+\frac{3}{2}\right)_{2} B_{\frac{1}{2},-\frac{3}{2}}, \quad\left(\delta+\frac{3}{2}\right)_{3} B_{\frac{3}{2},-\frac{3}{2}} .
$$

The equivalence question in length 4 at $n=0$ and $n=-2$ is resolved by the following proposition. 
Proposition 4.2. For $l=4$ and $n=0, \mathrm{SQ}_{\lambda, \mu}^{\delta, 4}$ and $\mathrm{SQ}_{\lambda^{\prime}, \mu^{\prime}}^{\delta^{\prime}, 4}$ are equivalent if and only if they induce simultaneous vanishing of

$$
\delta \gamma^{1 / 2}, \quad(\delta)_{2} B_{2,0}, \quad(\delta-1)_{2} B_{3,1},
$$

and if $(\delta-1)_{2} B_{3,1}=0$, induce also simultaneous vanishing of $(\delta)_{4} \gamma^{1 / 2}$.

In the dual case $l=4$ and $n=-2, \mathrm{SQ}_{\lambda, \mu}^{\delta+2,4}$ and $\mathrm{SQ}_{\lambda^{\prime}, \mu^{\prime}}^{\delta^{\prime}+2,4}$ are equivalent if and only if they induce simultaneous vanishing of

$$
\delta \gamma^{1 / 2}, \quad(\delta+1)_{2} B_{1,-1}, \quad(\delta+2)_{2} B_{0,-2},
$$

and if $(\delta+2)_{2} B_{0,-2}=0$, induce also simultaneous vanishing of $(\delta+3)_{4} \gamma^{1 / 2}$.

Note that in the $n=0$ differential operator case $\mathrm{SQ}_{\lambda, \mu}^{3,4}=\mathcal{D}_{\lambda, \lambda+3}^{3}$, the additional condition is automatically satisfied because $\delta=3$. This is why our results agree with those of Ga00] here.

Last we treat the self-dual cases in lengths 4 and 5 . The self-dual $l=4$ case at $n=-1$ is particularly interesting because there is a new rational invariant:

$$
R(\gamma, \delta):=\frac{\gamma^{1 / 2} B_{2,-1}}{B_{2,0} B_{1,-1}}=\frac{\gamma(\gamma-3)}{(\gamma-\delta-1)(\gamma+\delta-1)} .
$$

Theorem 4.3. If $\mathrm{SQ}_{\lambda, \mu}^{\delta+1,4}$ and $\mathrm{SQ}_{\lambda^{\prime}, \mu^{\prime}}^{\delta^{\prime}+1,4}$ are equivalent, then they satisfy the $S V C$, that is, they induce simultaneous vanishing of

$$
\delta \gamma^{1 / 2}, \quad(\delta+1)_{3} \gamma^{1 / 2}(\gamma-3), \quad(\delta)_{2}(\gamma-\delta-1), \quad(\delta+1)_{2}(\gamma+\delta-1) .
$$

If at least one of these four functions does vanish on both subquotients, then simultaneous vanishing is sufficient for equivalence. If none of them vanish, then the subquotients are equivalent if and only if in addition $R(\gamma, \delta)=R\left(\gamma^{\prime}, \delta^{\prime}\right)$.

The level curves of $R$ comprise a pencil of conics which we shall describe in Section 6. This invariant is the reason for the exceptional behaviour of the modules $\mathcal{D}_{\lambda, \lambda+2}^{3}=\mathrm{SQ}_{\lambda, \lambda+2}^{3,4}$ pointed out in Theorem 3.3(4) and Section 10.1 of [Ga00]: at $\delta=2, R$ reduces to the monotonic function $\gamma /(\gamma+1)$, so each of these modules is equivalent only to its conjugate.

The self-dual $l=5$ case, where $n=-\frac{3}{2}$, is exceptional for the opposite reason: it is simpler than the other $l=5$ resonant cases. We do not treat any other resonant $l \geq 5$ cases in this article, but one could do so using the simplified formulas given in Section 7 for the coefficients $\bar{b}_{m, n}$ first studied in [CS04].

Proposition 4.4. The subquotients $\mathrm{SQ}_{\lambda, \mu}^{\delta+\frac{3}{2}, 5}$ and $\mathrm{SQ}_{\lambda^{\prime}, \mu^{\prime}}^{\delta^{\prime}+\frac{3}{2}, 5}$ are equivalent if and only if they satisfy the conditions of Theorem 3.4 with $n=-\frac{3}{2}$.

\section{LACUNARY SUBQUOTIENTS}

In this section we answer the equivalence question in certain non-resonant lacunary cases. Again, proofs are deferred to Section 7 It has long been known that for $k \neq \delta, \Psi_{\lambda, \mu}^{k}$ has a unique lacunary $\operatorname{Vect}(\mathbb{R})$-invariant submodule

$$
\Psi_{\lambda, \mu}^{k, \text { lac }}, \text { with composition series }\left\{\mathcal{F}_{\delta-k}, \mathcal{F}_{\delta-k+2}, \mathcal{F}_{\delta-k+3}, \mathcal{F}_{\delta-k+4}, \ldots\right\} \text {. }
$$

A proof may be found in CO05], where $\Psi_{\lambda, \mu}^{k, \text { lac }}$ is called $\Psi_{1}^{k}(\lambda, \delta)$. The module is given by applying the $(\lambda, \mu)$-projective quantization (see Section 17) to $\mathcal{F}_{\delta-k} \oplus$ $\bigoplus_{i=2}^{\infty} \mathcal{F}_{\delta-k+i}$. The next two propositions answer the equivalence question for the simplest lacunary subquotients; they are parallel to Propositions 3.1 and 3.2 . 
Proposition 5.1. The subquotients $\Psi_{\lambda, \mu}^{\delta-n \text { lac }} / \Psi_{\lambda, \mu}^{\delta-n-3}$ and $\Psi_{\lambda^{\prime}, \mu^{\prime}}^{\delta^{\prime}-n \text { lac }} / \Psi_{\lambda^{\prime}, \mu^{\prime}}^{\delta^{\prime}-n}$ have composition series $\left\{\mathcal{F}_{n}, \mathcal{F}_{n+2}\right\}$. For $n \neq 0$, i.e., $N_{3} \neq \frac{1}{2}$, they are equivalent if and only if they induce simultaneous vanishing of (3).

The subquotients $\Psi_{\lambda, \mu}^{\delta-n} / \Psi_{\lambda, \mu}^{\delta-n-1, \text { lac }}$ and $\Psi_{\lambda^{\prime}, \mu^{\prime}}^{\delta^{\prime}} / \Psi_{\lambda^{\prime}, \mu^{\prime}}^{\delta^{\prime}-n-1 \text { lac }}$ also have composition series $\left\{\mathcal{F}_{n}, \mathcal{F}_{n+2}\right\}$. For $n \neq-1$, i.e., $N_{3} \neq-\frac{1}{2}$, they too are equivalent if and only if they induce simultaneous vanishing of (3). The two cases are dual.

Proposition 5.2. The subquotients $\Psi_{\lambda, \mu}^{\delta-n \text {,lac }} / \Psi_{\lambda, \mu}^{\delta-n-4}$ and $\Psi_{\lambda^{\prime}, \mu^{\prime}}^{\delta^{\prime}-\text { lac }} / \Psi_{\lambda^{\prime}, \mu^{\prime}}^{\delta^{\prime}-n}$ have composition series $\left\{\mathcal{F}_{n}, \mathcal{F}_{n+2}, \mathcal{F}_{n+3}\right\}$. For $n \notin\{-2,0\}$, i.e., $N_{4} \neq \pm 1$, they are equivalent if and only if they induce simultaneous vanishing of

$$
\left(\delta-N_{4}+1\right)_{2} B_{n+2, n}, \quad\left(\delta-N_{4}+1\right)_{3} B_{n+3, n} .
$$

The subquotients $\Psi_{\lambda, \mu}^{\delta-n} / \Psi_{\lambda, \mu}^{\delta-n-2 \text {,lac }}$ and $\Psi_{\lambda^{\prime}, \mu^{\prime}}^{\delta^{\prime}-n} / \Psi_{\lambda^{\prime}, \mu^{\prime}}^{\delta^{\prime}-n \text {, lac }}$ have composition series $\left\{\mathcal{F}_{n}, \mathcal{F}_{n+1}, \mathcal{F}_{n+3}\right\}$, dual to the first case. For $n \notin\{-2,0\}$, i.e., $N_{4} \neq \pm 1$, they are equivalent if and only if they induce simultaneous vanishing of

$$
\left(\delta-N_{4}\right)_{2} B_{n+3, n+1}, \quad\left(\delta-N_{4}+1\right)_{3} B_{n+3, n} .
$$

The first case in which both the numerator and the denominator of the subquotient are lacunary has composition series $\left\{\mathcal{F}_{n}, \mathcal{F}_{n+2}, \mathcal{F}_{n+4}\right\}$. Here we obtain a single rational invariant, the function $I_{n}$ arising in Section 3.3 .

Theorem 5.3. The subquotients $\Psi_{\lambda, \mu}^{\delta-n \text {, lac }} / \Psi_{\lambda, \mu}^{\delta-n-3 \text {,lac }}$ and $\Psi_{\lambda^{\prime}, \mu^{\prime}}^{\delta^{\prime}-n \text { lac }} / \Psi_{\lambda^{\prime}, \mu^{\prime}}^{\delta^{\prime}-n-3 \text {, lac }}$ have composition series $\left\{\mathcal{F}_{n}, \mathcal{F}_{n+2}, \mathcal{F}_{n+4}\right\}$. For

$$
n \notin\left\{-3,-\frac{5}{2},-\frac{1}{2}, 0\right\}, \text { i.e., } N_{5} \notin\left\{ \pm 1, \pm \frac{3}{2}\right\},
$$

if they are equivalent, then they induce simultaneous vanishing of

$$
\left(\delta-N_{5}+\frac{3}{2}\right)_{2} B_{n+2, n}, \quad\left(\delta-N_{5}-\frac{1}{2}\right)_{2} B_{n+4, n+2}, \quad\left(\delta-N_{5}+\frac{3}{2}\right)_{4} B_{n+4, n} .
$$

If at least one of these three functions does vanish on both subquotients, then simultaneous vanishing is sufficient for equivalence. If none of them vanish, then the subquotients are equivalent if and only if in addition $I_{n}(\gamma, \delta)=I_{n}\left(\gamma^{\prime}, \delta^{\prime}\right)$.

Thus generically, subquotients with composition series $\left\{\mathcal{F}_{n}, \mathcal{F}_{n+2}, \mathcal{F}_{n+4}\right\}$ are equivalent if and only if their parameters in $(\gamma, \delta)$-space lie on the same level curve of $I_{n}$. As we mentioned in Section 3.3, these level curves comprise a pencil of conics which is described in Section 6 .

For completeness, we also answer the equivalence question for the two other lacunary composition series beginning with $\mathcal{F}_{n}$ and ending with $\mathcal{F}_{n+4}$ :

Corollary 5.4. The subquotients $\Psi_{\lambda, \mu}^{\delta-n \text { lac }} / \Psi_{\lambda, \mu}^{\delta-n-5}$ and $\Psi_{\lambda^{\prime}, \mu^{\prime}}^{\delta^{\prime}-n} / \Psi_{\lambda^{\prime}, \mu^{\prime}}^{\delta^{\prime}-n-5}$ have composition series $\left\{\mathcal{F}_{n}, \mathcal{F}_{n+2}, \mathcal{F}_{n+3}, \mathcal{F}_{n+4}\right\}$. Under the same restriction on $n$ as in Theorem 5.3, they are equivalent if and only if they satisfy the conditions of Theorem 5.3 and in addition induce simultaneous vanishing of $\left(\delta-N_{5}+\frac{3}{2}\right)_{3} B_{n, n+3}$.

The subquotients $\Psi_{\lambda, \mu}^{\delta-n} / \Psi_{\lambda, \mu}^{\delta-n-3 \text {,lac }}$ and $\Psi_{\lambda^{\prime}, \mu^{\prime}}^{\delta^{\prime}-n} / \Psi_{\lambda^{\prime}, \mu^{\prime}}^{\delta^{\prime}-n-5}$ have composition series $\left\{\mathcal{F}_{n}, \mathcal{F}_{n+1}, \mathcal{F}_{n+2}, \mathcal{F}_{n+4}\right\}$, dual to the last case. Still under the same restriction on $n$, they are equivalent if and only if they satisfy the conditions of Theorem 5.3 and in addition induce simultaneous vanishing of $\left(\delta-N_{5}+\frac{1}{2}\right)_{3} B_{n+1, n+4}$. 
In the case of the composition series $\left\{\mathcal{F}_{n}, \mathcal{F}_{n+2}, \mathcal{F}_{n+3}, \mathcal{F}_{n+5}\right\}$ we obtain a new rational invariant. In order to make the symmetry of Corollary 2.4 transparent, we express the relevant functions $B_{n+i, n}$ in terms of $N_{6}$ :

$$
\begin{aligned}
B_{n+2, n} & =\gamma-\left[\left(2 N_{6}-3\right) \delta+\left(N_{6}^{2}-3 N_{6}+3\right)\right], \\
B_{n+5, n+3} & =\gamma-\left[\left(2 N_{6}+3\right) \delta+\left(N_{6}^{2}+3 N_{6}+3\right)\right], \\
B_{n+3, n} & =\gamma^{3 / 2}-3 \gamma^{1 / 2}\left[\left(N_{6}-1\right) \delta+1\right], \\
B_{n+5, n+2} & =\gamma^{3 / 2}-3 \gamma^{1 / 2}\left[\left(N_{6}+1\right) \delta+1\right] .
\end{aligned}
$$

The invariant is

$$
M_{n}(\gamma, \delta):=B_{n+5, n+2} B_{n+2, n} / B_{n+5, n+3} B_{n+3, n} .
$$

Theorem 5.5. The subquotients $\Psi_{\lambda, \mu}^{\delta-n \text {, lac }} / \Psi_{\lambda, \mu}^{\delta-n-4, \text { lac }}$ and $\Psi_{\lambda^{\prime}, \mu^{\prime}}^{\delta^{\prime}-n \text { lac }} / \Psi_{\lambda^{\prime}, \mu^{\prime}}^{\delta^{\prime}-n-4 \text {, lac }}$ have composition series $\left\{\mathcal{F}_{n}, \mathcal{F}_{n+2}, \mathcal{F}_{n+3}, \mathcal{F}_{n+5}\right\}$. For

$$
n \notin\left\{-4,-\frac{7}{2},-3,-2,-1,-\frac{1}{2}, 0\right\}, \quad \text { i.e., } N_{6} \notin\left\{0, \pm 1, \pm \frac{3}{2}, \pm 2\right\},
$$

if they are equivalent, then they induce simultaneous vanishing of

$$
\begin{array}{ll}
\left(\delta-N_{6}+2\right)_{2} B_{n+2, n}, & \left(\delta-N_{6}-1\right)_{2} B_{n+5, n+3}, \\
\left(\delta-N_{6}+2\right)_{3} B_{n+3, n}, & \left(\delta-N_{6}\right)_{3} B_{n+5, n+2 .} .
\end{array}
$$

If at least one of these four functions does vanish on both subquotients, then simultaneous vanishing is sufficient for equivalence. If none of them vanish, then the subquotients are equivalent if and only if in addition $M_{n}(\gamma, \delta)=M_{n}\left(\gamma^{\prime}, \delta^{\prime}\right)$.

Note that when $\gamma \neq 0$, the $\gamma^{1 / 2}$ factors of $M_{n}$ cancel, so its level curves comprise a new pencil of conics. This pencil too will be described in Section 6 .

The analog of Corollary 5.4 here involves both $I_{n}$ and $M_{n}$ :

Theorem 5.6. The subquotients $\Psi_{\lambda, \mu}^{\delta-n, \mathrm{lac}} / \Psi_{\lambda, \mu}^{\delta-n-6}$ and $\Psi_{\lambda^{\prime}, \mu^{\prime}}^{\delta^{\prime}-n, \mathrm{lac}} / \Psi_{\lambda^{\prime}, \mu^{\prime}}^{\delta^{\prime}-n-6}$ have composition series $\left\{\mathcal{F}_{n}, \mathcal{F}_{n+2}, \mathcal{F}_{n+3}, \mathcal{F}_{n+4}, \mathcal{F}_{n+5}\right\}$. For

$$
n \notin\left\{-4,-\frac{7}{2},-3,-2,-1,-\frac{1}{2}, 0\right\},
$$

they are equivalent if and only if they satisfy all of the following conditions:

(i) They induce simultaneous vanishing of (5) for

$$
(i, j) \in\{(2,0),(4,2),(5,3),(3,0),(5,2),(4,0)\} .
$$

(ii) If (5) is non-zero for $(i, j) \in\{(2,0),(4,2),(4,0)\}$, then

$$
I_{n}(\gamma, \delta)=I_{n}\left(\gamma^{\prime}, \delta^{\prime}\right)
$$

(iii) If (5) is non-zero for $(i, j) \in\{(2,0),(5,3),(3,0),(5,2)\}$, then

$$
M_{n}(\gamma, \delta)=M_{n}\left(\gamma^{\prime}, \delta^{\prime}\right) \text {. }
$$

The subquotients $\Psi_{\lambda, \mu}^{\delta-n} / \Psi_{\lambda, \mu}^{\delta-n-4, \text { lac }}$ and $\Psi_{\lambda^{\prime}, \mu^{\prime}}^{\delta^{\prime}-n} / \Psi_{\lambda^{\prime}, \mu^{\prime}}^{\delta^{\prime}-n-4 \text {, lac }}$ have composition series $\left\{\mathcal{F}_{n}, \mathcal{F}_{n+1}, \mathcal{F}_{n+2}, \mathcal{F}_{n+3}, \mathcal{F}_{n+5}\right\}$. They are equivalent if and only if the dual subquotients $\Psi_{\mu, \lambda}^{n+4-\delta \text {,lac }} / \Psi_{\mu, \lambda}^{n-2-\delta}$ and $\Psi_{\mu^{\prime}, \lambda^{\prime}}^{n+4-\delta^{\prime}, \text { lac }} / \Psi_{\mu^{\prime}, \lambda^{\prime}}^{n-2-\delta^{\prime}}$ are equivalent.

Thus generically, subquotients with composition series $\left\{\mathcal{F}_{n}, \mathcal{F}_{n+2}, \mathcal{F}_{n+3}, \mathcal{F}_{n+4}\right.$, $\left.\mathcal{F}_{n+5}\right\}$ are equivalent if and only if their parameters in $(\gamma, \delta)$-space lie on the same level curves of both $I_{n}$ and $M_{n}$. Since both level curves are conics, the equivalence classes usually consist of four conjugate pairs of subquotients. 


\section{Equivalence PENCILS}

In this section we examine the level curves in $(\gamma, \delta)$-space of some of the rational invariants occurring in Theorems 3.4, 4.3, 5.3, 5.5, and 5.6.

6.1. The invariant $R=\gamma^{1 / 2} B_{2,-1} / B_{2,0} B_{1,-1}$. Together with the SVC, $R$ is a complete invariant for the equivalence classes $\mathrm{EC}_{-1}^{4}$ of length 4 subquotients with self-dual composition series given in Theorem 4.3. It is more convenient to work with the invariant

$$
\tilde{R}:=\frac{R}{1-R}=\frac{\gamma^{2}-3 \gamma}{\gamma+1-\delta^{2}},
$$

which of course is also complete. Its level curves form the pencil of conics passing through the four points $(0, \pm 1)$ and $(3, \pm 2)$, the simultaneous zeroes of the numerator and denominator. The conic at level $\tilde{R}$ may be written as

$$
\left[\gamma-\frac{1}{2}(\tilde{R}-3)\right]^{2}+\tilde{R} \delta^{2}=\frac{1}{4}(\tilde{R}+1)(\tilde{R}+9) .
$$

For $\tilde{R}$ real, the real points form ellipses for $\tilde{R}>0$, hyperbolas for $\tilde{R}<0$, a parabola for $\tilde{R}=\infty$, two parallel lines for $\tilde{R}=0$, and two crossing lines for $\tilde{R}=-1$ or -9 . These different zones are delineated by shadings in Figure 1 .

$\delta$

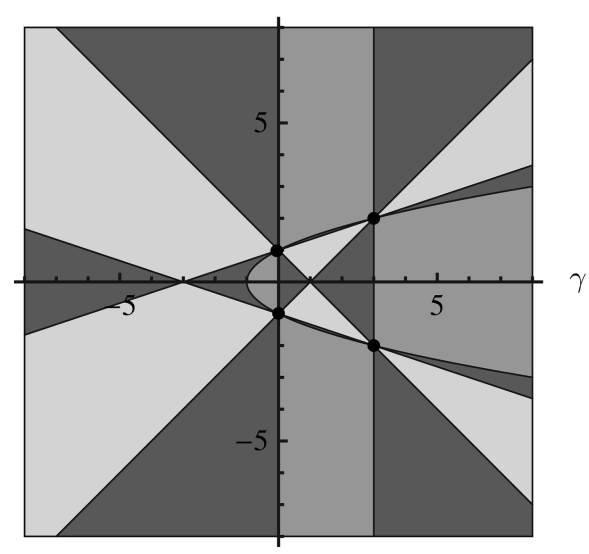

$\delta$

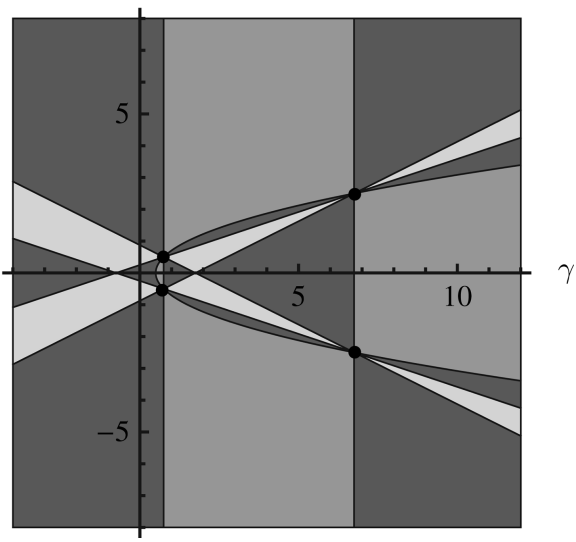

FiguRE 1. These are self-dual resonant invariants: at left is $R$ and at right is $I_{-3 / 2}$. Depicted are the four points determining the pencil of conics and the lines and parabola bordering the different zones. The zones of up-down and right-left hyperbolas are shaded light and dark, respectively, and the zone of ellipses is shaded grey.

6.2. The invariant $I_{n}=B_{n+4, n} / B_{n+4, n+2} B_{n+2, n}$. This is the simpler of the two continuous invariants for the equivalence classes $\mathrm{EC}_{n}^{5}$ of length 5 subquotients treated in Theorem 3.4 and Proposition 4.4 and together with the SVC it is a complete invariant for the equivalence classes of lacunary modules treated in Theorem 5.3. Like $R$, its level curves form a pencil of conics. To describe them it will 
be convenient to use the coordinates $\left(\tilde{\gamma}_{5}, \delta\right)$, where $\tilde{\gamma}_{5}:=\gamma-2 N_{5} \delta$, as the conics are all in standard orientation in these coordinates. Some computation gives

$$
\begin{aligned}
& B_{n+3 \pm 1, n+1 \pm 1}=\left[\tilde{\gamma}_{5}-N_{5}^{2}-\frac{7}{4}\right] \mp 2\left[\delta+N_{5}\right], \\
& B_{n+4, n}=\left[\tilde{\gamma}_{5}+N_{5}^{2}-\frac{15}{4}\right]^{2}-4\left[N_{5} \delta-\frac{1}{5} N_{5}^{2}+\frac{6}{5}\right]^{2}-\frac{9}{25}\left(N_{5}^{2}-1\right)\left(4 N_{5}^{2}-9\right) .
\end{aligned}
$$

The pencil of conics is determined by the four simultaneous zeroes of $B_{n+4, n}$ and $B_{n+4, n+2} B_{n+2, n}$. In $\left(\tilde{\gamma}_{5}, \delta\right)$-coordinates, these are

$$
\left(N_{5}^{2} \pm 4 N_{5}+\frac{27}{4}, N_{5} \pm \frac{5}{2}\right), \quad\left(N_{5}^{2} \pm \frac{4}{5} N_{5}+\frac{3}{4},-\frac{3}{5} N_{5} \mp \frac{1}{2}\right)
$$

a quadrilateral with center $\left(N_{5}^{2}+\frac{15}{4}, \frac{1}{5} N_{5}\right)$. The slopes of the six lines connecting the vertices are $\pm 2, \pm 3$, and $\pm \frac{8}{5} N_{5}$, from which it follows that the quadrilateral is cyclic (inscribed). Note that at $N_{5}=0$ and as $N_{5} \rightarrow \infty$ it becomes a trapezoid. When $N_{5}$ is $\pm 5 / 4$ or $\pm 15 / 8$ two of the four points coincide; these are the only values of $N_{5}$ for which this occurs.

We remark that since $B_{n+4, n}$ is a conic, it is at first surprising that the four vertices are all rational in $N_{5}$. However, this is explained by the final paragraphs of Co05]: one of the two simultaneous zeroes of $B_{n+2, n}$ and $B_{n+4, n}$ is also a zero of $B_{n+3, n} / \gamma^{1 / 2}$, a linear function, so it is rational, forcing the other to be rational. The rationality of the simultaneous zeroes of $B_{n+4, n+2}$ and $B_{n+4, n}$ follows by duality.

Completing the square in $\tilde{\gamma}_{5}$ and $\delta$, a long calculation gives the following form of the conic at level $I_{n}$ :

$$
\begin{aligned}
& \left(I_{n}-N_{5}^{2}\right)\left[\left(I_{n}-1\right)\left(\tilde{\gamma}_{5}-N_{5}^{2}-\frac{15}{4}\right)+2\left(I_{n}-N_{5}^{2}\right)\right]^{2} \\
& -4\left(I_{n}-1\right)\left[\left(I_{n}-N_{5}^{2}\right)\left(\delta-\frac{1}{5} N_{5}\right)+\frac{6}{5}\left(I_{n}-1\right) N_{5}\right]^{2} \\
= & -\frac{1}{25}\left(N_{5}^{2}-1\right)\left[9\left(I_{n}-1\right)-4\left(I_{n}-N_{5}^{2}\right)\right]\left[16 N_{5}^{2}\left(I_{n}-1\right)-25\left(I_{n}-N_{5}^{2}\right)\right] .
\end{aligned}
$$

When $I_{n}$ is 1 or $N_{5}^{2}$, taking limits in the obvious way gives a parabola. We obtain ellipses when $\left(I_{n}-1\right)\left(I_{n}-N_{5}^{2}\right)$ is negative and hyperbolas when it is positive. When $I_{n}$ is $\infty,-\frac{4}{5}\left(N_{5}^{2}-\frac{9}{4}\right)$, or $-9 N_{5}^{2} /\left(16 N_{5}^{2}-25\right)$, the right side is zero and we obtain a degenerate hyperbola: either a pair of opposite sides of the quadrilateral or its diagonal.

These zones may be seen for some values of $n$ in Figures 1, 2, and 3 , The zone of vertical hyperbolas is light, the zone of horizontal hyperbolas is dark, and the zone of ellipses is grey. Some simple rules describe the zones: crossing either of the two parabolas toggles between the elliptical zone and the hyperbolic zones, and crossing a line toggles between the two hyperbolic zones. Crossing over a parabola out of a hyperbolic zone and then back over the same parabola leads to the same hyperbolic zone, whereas crossing back over the other parabola leads to the other hyperbolic zone.

On the right in Figure 1 is the self-dual case $I_{-3 / 2}$ relevant to Proposition 4.4, where $N_{5}=0$. In Figure 2 we show the resonant case $I_{-1}$ occurring in Theorem 5.3 , where $N_{5}=1 / 2$, and the case $I_{-1 / 4}$, where $N_{5}=5 / 4$ and the quadrilateral has a double vertex, causing two of the degenerate hyperbolas to coincide. On the left in Figure 3 is $I_{10.5}$, where one can see the horizontal parabola approaching a pair of lines. In all cases the magnification is the same on both axes, but in the non-selfdual cases we have not numbered the $\tilde{\gamma}_{5}$ axis because it is shifted in order to center on the quadrilateral. Observe that the rules describing the zones sometimes appear 
to be violated, because some of the zones are too thin to be seen in the figures. For example, in the picture of $I_{-1}$ the line and the vertical parabola connecting the two left points cannot be distinguished, so the light zone between them is invisible.
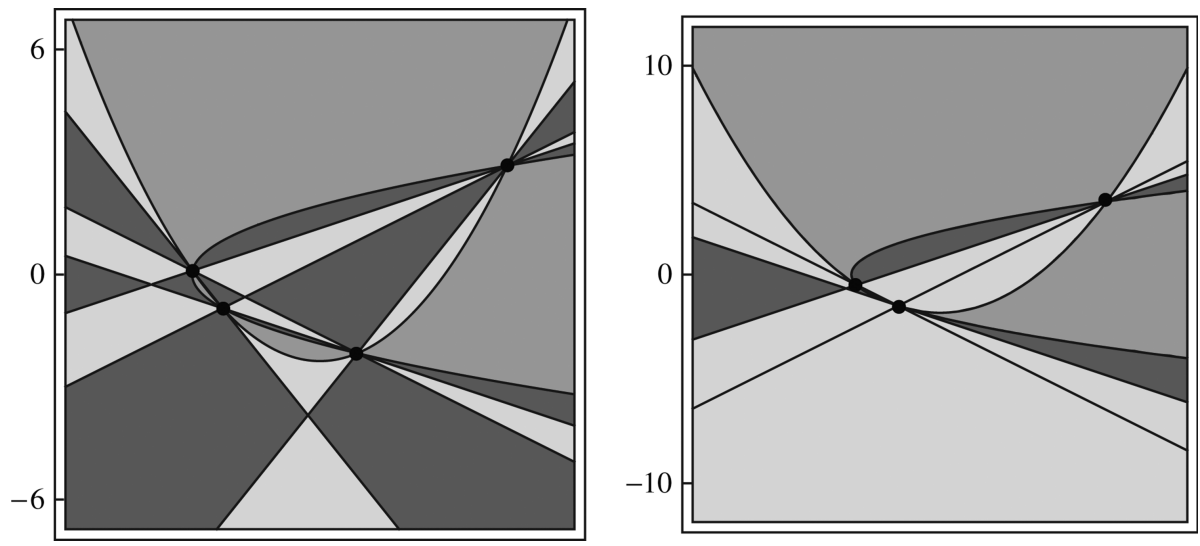

Figure 2. Zones of ellipses and hyperbolas for $I_{-1}$ (left) and $I_{-1 / 4}$ (right) in $\left(\tilde{\gamma}_{5}, \delta\right)$-coordinates. The zones are shaded as in Figure 1 .

Recall from Theorem 5.3 that $I_{n}$ is meaningless when $N_{5}$ is one of the resonant values \pm 1 or $\pm \frac{3}{2}$. The values $N_{5}= \pm 1$ are particularly special: there $B_{n+4, n}$ and $B_{n+4, n+2} B_{n+2, n}$ are equal by Corollary 7.12 , so $I_{n}$ is identically 1 . It follows that $B_{n+4, n}-B_{n+4, n+2} B_{n+2, n}$ is divisible by $N_{5}^{2}-1$, so we define

$$
B_{420}:=\frac{B_{n+4, n}-B_{n+4, n+2} B_{n+2, n}}{4\left(N_{5}^{2}-1\right)}=\gamma-\left[\delta^{2}+\frac{8}{5} N_{5} \delta+\frac{2}{5} N_{5}^{2}+\frac{1}{2}\right] .
$$

Just as we replaced the invariant $R$ by $\tilde{R}$, we can replace $I_{n}$ by

$$
\tilde{I}_{n}:=\frac{4\left(N_{5}^{2}-1\right)}{I_{n}-1}=\frac{B_{n+4, n+2} B_{n+2, n}}{B_{420}} .
$$

Where $I_{n}$ alone is concerned, this simplifies some calculations, but not the final result (77). However, it is a useful idea when considering $I_{n}$ together with $J_{n}$, which we now do.

6.3. The invariants $I_{n}$ and $J_{n}=B_{n+4, n} B_{n+3, n+1} / B_{n+4, n+1} B_{n+3, n}$. By Theorem 3.4 and Proposition 4.4, these two invariants together with the SVC completely classify the equivalence classes $\mathrm{EC}_{n}^{5}$ unless $N_{5}$ takes on one of the resonant values $\pm \frac{1}{2}, \pm 1$, and $\pm \frac{3}{2}$. Applying Corollary 7.12 , we find that $J_{n}$ is identically 1 for $N_{5}= \pm \frac{3}{2}$; for example, at $N_{5}=\frac{3}{2}$ we have $n=0, B_{4,0}=\gamma^{1 / 2} B_{4,1}$, and $B_{3,0}=\gamma^{1 / 2} B_{3,1}$. Hence the difference $B_{n+4, n} B_{n+3, n+1}-B_{n+4, n+1} B_{n+3, n}$ is divisible by $N_{5}^{2}-\frac{9}{4}$. We define

$$
\begin{aligned}
& B_{4310}:=\frac{B_{n+4, n} B_{n+3, n+1}-B_{n+4, n+1} B_{n+3, n}}{N_{5}^{2}-\frac{9}{4}} \\
& =\gamma^{2}-\gamma\left[\delta^{2}+\frac{12}{5} N_{5} \delta+\frac{13}{5} N_{5}^{2}+\frac{3}{4}\right]+\frac{3}{5}\left[2 N_{5} \delta+N_{5}^{2}+\frac{3}{4}\right]\left[4 N_{5} \delta+N_{5}^{2}+\frac{15}{4}\right] .
\end{aligned}
$$


Now combine $B_{420}$ and $B_{4310}$ in the following two ways: set

$$
\begin{aligned}
& B_{43210}^{-}:=5\left(B_{4310}-B_{n+3, n+1} B_{420}\right) \\
& =2 \gamma\left[3 N_{5} \delta-3 N_{5}^{2}+\frac{5}{4}\right]-\left[2 N_{5} \delta+N_{5}^{2}+\frac{3}{4}\right]\left[5 \delta^{2}-4 N_{5} \delta-N_{5}^{2}-\frac{35}{4}\right], \\
& B_{43210}^{+}:=\frac{1}{2}\left(B_{4310}+B_{n+3, n+1} B_{420}\right) \\
& =\gamma^{2}-\gamma\left[\delta^{2}+3 N_{5} \delta+2 N_{5}^{2}+1\right]+\frac{1}{2}\left[2 N_{5} \delta+N_{5}^{2}+\frac{3}{4}\right]\left[\delta^{2}+4 N_{5} \delta+N_{5}^{2}+\frac{11}{4}\right] .
\end{aligned}
$$

We will prove the following proposition at the end of Section 7.1 . Define

$$
\tilde{J}_{n}:=B_{43210}^{+} / B_{43210}^{-} .
$$

Proposition 6.1. For any linearly independent elements $x$ and $y$ of $\mathbb{C}^{3}$,

$$
\frac{x_{1} B_{n+4, n+1} B_{n+3, n}+x_{2} B_{n+4, n} B_{n+3, n+1}+x_{3} B_{n+4, n+2} B_{n+3, n+1} B_{n+2, n}}{y_{1} B_{n+4, n+1} B_{n+3, n}+y_{2} B_{n+4, n} B_{n+3, n+1}+y_{3} B_{n+4, n+2} B_{n+3, n+1} B_{n+2, n}}
$$

is invariant under equivalence. In particular, $\tilde{J}_{n}$ is an invariant. In the situation of Part (v) of Theorem [3.4, $\tilde{I}_{n}$ and $\tilde{J}_{n}$ form a complete set of invariants.

We write $\tilde{I}_{n}$ and $\tilde{J}_{n}$ together for reference:

$$
\begin{aligned}
& \tilde{I}_{n}=\frac{\gamma^{2}-2 \gamma\left[2 N_{5} \delta+N_{5}^{2}+\frac{7}{4}\right]+\left[\left(2 N_{5} \delta+N_{5}^{2}+\frac{7}{4}\right)^{2}-4\left(\delta+N_{5}\right)^{2}\right]}{\gamma-\left[\delta^{2}+\frac{8}{5} N_{5} \delta+\frac{2}{5} N_{5}^{2}+\frac{1}{2}\right]}, \\
& \tilde{J}_{n}=\frac{\gamma^{2}-\gamma\left[\delta^{2}+3 N_{5} \delta+2 N_{5}^{2}+1\right]+\frac{1}{2}\left[2 N_{5} \delta+N_{5}^{2}+\frac{3}{4}\right]\left[\delta^{2}+4 N_{5} \delta+N_{5}^{2}+\frac{11}{4}\right]}{2 \gamma\left[3 N_{5} \delta-3 N_{5}^{2}+\frac{5}{4}\right]-\left[2 N_{5} \delta+N_{5}^{2}+\frac{3}{4}\right]\left[5 \delta^{2}-4 N_{5} \delta-N_{5}^{2}-\frac{35}{4}\right]} .
\end{aligned}
$$

Let us remark that eliminating $\gamma$ from these equations generically yields a sextic in $\delta$ with coefficients depending on $N_{5}, \tilde{I}_{n}$, and $\tilde{J}_{n}$ : simply clear denominators in both equations, take the difference to eliminate $\gamma^{2}$, solve for $\gamma$ in terms of $\delta$, plug the result into the formula for $\tilde{I}_{n}$, and clear denominators again. As we observed at the end of Section 3, this is expected: each equivalence class $\operatorname{EC}_{n}^{5}(\gamma, \delta)$ is the intersection of a conic and a cubic, so generic classes contain six points.

We have not depicted the family of cubics involved because it is not unique. The family of conics is necessarily the set of level curves of $I_{n}$, which is the same as the set of level curves of $\tilde{I}_{n}$, but the family of cubics can be altered by adding multiples of $I_{n}$ to the cubic invariant. It is always a pencil determined by nine points, but there is a 1-parameter family of choices for this set of points, and we did not find any "best" choice. For example, $J_{n}, K_{n}$, and $\tilde{J}_{n}$ all yield different choices.

We now compare our results in length 5 with those of [LO99] and Ga00. Recall that [O99] treats only those subquotients of pseudodifferential operators with $\lambda=\mu$, i.e., $\delta=0$. In fact a further restriction is imposed: only subquotients of real order $k$ are admitted, so $n$ and hence $N_{5}$ must be real. The following result is stated: for $k$ real and not non-negative half-integral, the subquotients $\mathrm{SQ}_{\lambda, \lambda}^{k, 5}$ and $\mathrm{SQ}_{\lambda^{\prime}, \lambda^{\prime}}^{k, 5}$ are equivalent if and only if they are either equal or conjugate; i.e., $\lambda^{\prime}$ is either $\lambda$ or $1-\lambda$.

On the other hand, as we have seen, Ga00. allows $\lambda$ and $\mu$ to vary independently but treats only differential operator modules. In fact, both $\lambda$ and $\mu$ are required to be real, so again, $n$ and $N_{5}$ must be real. The following result is stated: for $\lambda$ and $\mu$ real, the differential operator modules $\mathcal{D}_{\lambda, \mu}^{4}$ and $\mathcal{D}_{\lambda^{\prime}, \mu^{\prime}}^{4}$ are equivalent if and only if they are either equal or conjugate. 
Let us use Theorem 3.4 to generalize these results in the non-resonant cases. We begin with the Ga00 result, where the analysis is simpler. The statement is true for all complex values of $\lambda$ and $\mu$.

Proposition 6.2 (Ga00). Two non-resonant modules $\mathcal{D}_{\lambda, \mu}^{4}$ and $\mathcal{D}_{\lambda^{\prime}, \mu^{\prime}}^{4}$ are equivalent if and only if they are either equal or conjugate; i.e., $\left(\lambda^{\prime}, \mu^{\prime}\right)$ is either $(\lambda, \mu)$ or $(1-\mu, 1-\lambda)$.

Proof. The order $k=\delta-n$ is 4 at $\delta=N_{5}+\frac{5}{2}$. Evaluating $\tilde{J}_{n}$ here (we used a software package) gives

$$
\frac{2 \gamma-\left(N_{5}+1\right)\left(6 N_{5}+1\right)}{5\left(6 N_{5}+1\right)} .
$$

Thus at this value of $\delta$, the $\gamma$-linear denominator of the original expression for $\tilde{J}_{n}$ divides its $\gamma$-quadratic numerator for all values of $N_{5}$. (Probably there is a conceptual explanation of this.) Hence for $N_{5} \neq-\frac{1}{6}$, the invariant $\tilde{J}_{n}$ determines $\gamma$, proving the result. At $N_{5}=-\frac{1}{6}$, the alternate invariant $B_{4310} / B_{n+4, n+1} B_{n+3, n}$ reduces to $\gamma+\frac{5}{3}$, yielding a similar proof.

In the case of the LO99 result, our result is slightly different. We find that for most values of the pseudodifferential operator order $k$, exactly one equivalence class $\operatorname{EC}_{n}^{5}$ contains two points of the form $(\gamma, 0)$, and all the others contain only one. Thus if we fix $\delta$ at zero, then for most choices of the composition series, all but one of the subquotients is equivalent only to its conjugate, but one equivalence class consists of two conjugate pairs of subquotients.

To state the result concisely we make some preliminary definitions. Set

$$
\begin{array}{ll}
a_{I}:=-2\left(N_{5}^{2}+\frac{7}{4}\right), & b_{I}:=\left(N_{5}^{2}+2 N_{5}+\frac{7}{4}\right)\left(N_{5}^{2}-2 N_{5}+\frac{7}{4}\right), \\
c_{I}:=1, & d_{I}:=-\frac{2}{5}\left(N_{5}^{2}+\frac{5}{4}\right) \\
a_{J}:=-2\left(N_{5}^{2}+\frac{1}{2}\right), & b_{J}:=\frac{1}{2}\left(N_{5}^{2}+\frac{3}{4}\right)\left(N_{5}^{2}+\frac{11}{4}\right), \\
c_{J}:=-6\left(N_{5}^{2}-\frac{5}{12}\right), & d_{J}:=\left(N_{5}^{2}+\frac{3}{4}\right)\left(N_{5}^{2}+\frac{35}{4}\right),
\end{array}
$$

so that at $\delta=0$ we have

$$
\tilde{I}_{n}=\frac{\gamma^{2}+a_{I} \gamma+b_{I}}{c_{I} \gamma+d_{I}}, \quad \tilde{J}_{n}=\frac{\gamma^{2}+a_{J} \gamma+b_{J}}{c_{J} \gamma+d_{J}} .
$$

Define polynomials $f_{+}\left(N_{5}\right), f_{-}\left(N_{5}\right)$, and $E\left(N_{5}\right)$ by

$$
\begin{aligned}
f_{+} & :=-\frac{14}{5} N_{5}^{6}+\frac{38}{5} N_{5}^{4}+\frac{145}{8} N_{5}^{2}+\frac{175}{16}, \\
f_{-} & :=\frac{994}{25} N_{5}^{10}-\frac{5549}{20} N_{5}^{8}+322 N_{5}^{6}+\frac{29025}{32} N_{5}^{4}-\frac{12125}{128} N_{5}^{2}-\frac{380625}{1024}, \\
E & :=c_{I} d_{J}-c_{J} d_{I}=-\frac{7}{5}\left(N_{5}^{2}-\frac{25}{4}\right)\left(N_{5}^{2}+\frac{25}{28}\right) .
\end{aligned}
$$

Proposition 6.3. We assume that $N_{5}$ is not one of the resonant values $\pm \frac{1}{2}, \pm 1$, $\pm \frac{3}{2}$, and also that we are in the situation of Part (v) of Theorem [3.4. If $N_{5}$ is either a root of $f_{-}$or any of the values

$$
\pm \frac{5}{2}, \quad \pm \frac{5}{6}, \quad \pm \frac{i \sqrt{3}}{2}, \quad \pm \frac{5 i}{2 \sqrt{7}},
$$


then each equivalence class $\mathrm{EC}_{n}^{5}$ contains a unique element $(\gamma, 0)$ with $\delta$-coordinate zero.

At all other values of $N_{5}$, each equivalence class $\mathrm{EC}_{n}^{5}$ contains a unique element $(\gamma, 0)$ with $\delta$-coordinate zero with one exception: $(\gamma, 0)$ and $\left(\gamma^{\prime}, 0\right)$ are in the same equivalence class for the two values $\gamma$ and $\gamma^{\prime}$ determined by the equations

$$
\gamma+\gamma^{\prime}=f_{+} / E, \quad\left(\gamma-\gamma^{\prime}\right)^{2}=f_{-} / E^{2} .
$$

Proof. Use software to check that $\tilde{I}_{n}$ reduces to a linear polynomial in $\gamma$ if and only if $N_{5}^{2}$ is $\frac{25}{4}$ or $\frac{25}{36}$, and the same occurs for $\tilde{J}_{n}$ if and only if $N_{5}^{2}$ is $\frac{9}{4}, \frac{25}{4},-\frac{3}{4}$, or $-\frac{25}{28}$. At these values the proof goes as for Proposition 6.2

Now suppose that $N_{5}$ does not take on any of these values, and note that this implies $E \neq 0$. Under our assumptions, two values $\gamma$ and $\gamma^{\prime}$ give equivalent $\delta=0$ subquotients if and only if they give equal values of $\tilde{I}_{n}$ and $\tilde{J}_{n}$. Write this condition in terms of $\gamma_{ \pm}:=\gamma \pm \gamma^{\prime}$, clear denominators, assume that $\gamma_{-} \neq 0$, and divide by it. One obtains the equations in the proposition. The reader may check that the results are the same even at $N_{5}^{2}=\frac{5}{12}$, where $c_{J}=0$ so $\tilde{J}_{n}$ has $\gamma$-constant denominator.

We remark that the condition that the situation of Part (v) of Theorem 3.4 obtains could be sharpened to the condition that $N_{5}$ not be a root of any of a collection of non-trivial polynomials. We did not investigate the existence of double equivalence classes when $N_{5}$ is a root of one of these polynomials. It is easy to use our methods to analyze the situation for any given value of $N_{5}$, but analyzing all cases would be arduous and as far as we can see not interesting.

6.4. The invariant $M_{n}=B_{n+5, n+2} B_{n+2, n} / B_{n+5, n+3} B_{n+3, n}$. Together with the $\mathrm{SVC}$, this is a complete invariant for the lacunary equivalence classes treated in Theorem 5.5. Its level curves form a pencil of conics which we will only discuss briefly. Corollary 17.12 implies that at $N_{6}=0, B_{3,0} B_{0,-2}=B_{3,1} B_{1,-2}$, so we define $B_{5320}:=\frac{1}{6}\left(B_{n+5, n+2} B_{n+2, n}-B_{n+5, n+3} B_{n+3, n}\right) / N_{6}=\gamma^{3 / 2}-\gamma^{1 / 2}\left[\delta^{2}+2 N_{6} \delta+3\right]$.

Proceeding along the same lines as before, we replace $M_{n}$ by the invariant

$$
\tilde{M}_{n}:=\frac{6 N_{6} M_{n}}{M_{n}-1}=\frac{B_{n+5, n+2} B_{n+2, n}}{B_{5320}} .
$$

Setting $\tilde{\gamma}_{6}:=\gamma-\frac{5}{2} N_{6} \delta$, we find that the conics are all in standard orientation in the coordinates $\left(\tilde{\gamma}_{6}, \delta\right)$, as

$$
\tilde{M}_{n}:=\frac{\left[\tilde{\gamma}_{6}-\left(\frac{1}{2} N_{6}+3\right) \delta-3\right]\left[\tilde{\gamma}_{6}+\left(\frac{1}{2} N_{6}+3\right) \delta-\left(N_{6}^{2}-3 N_{6}+3\right)\right]}{\tilde{\gamma}_{6}-\delta^{2}+\frac{1}{2} N_{6} \delta-3} .
$$

Just as for $I_{n}$, the four simultaneous zeroes of the numerator and denominator determine the pencil of conics. In $\left(\tilde{\gamma}_{6}, \delta\right)$-coordinates, these zeroes are

$$
(3,0), \quad\left(-N_{6}^{2}+3,-N_{6}\right), \quad\left(\frac{1}{2} N_{6}^{2} \pm \frac{9}{2} N_{6}+12, N_{6} \pm 3\right) .
$$

The slopes of the six lines determined by this quadrilateral are $\pm 2 / 3$ and $\pm 2 /\left(N_{6} \pm+6\right)$. Thus the quadrilateral is cyclic and becomes a trapezoid at $N_{6}= \pm 6$ and as $N_{6} \rightarrow \infty$. Two of its vertices coincide when $N$ is $0, \pm \frac{3}{2}$, or \pm 3 ; the coincident vertices are at either $(3,0)$ or $\left(\frac{51}{8}, \pm \frac{3}{2}\right)$ in all cases.

The level curve of $\tilde{M}_{6}$ is a horizontal parabola at $\tilde{M}_{6}=\infty$ and a vertical parabola at $\tilde{M}_{6}=\left(\frac{1}{2} N_{6}+3\right)^{2}$. At $N_{6}= \pm 6$, where $n=4$ and -8 , the vertical parabola 

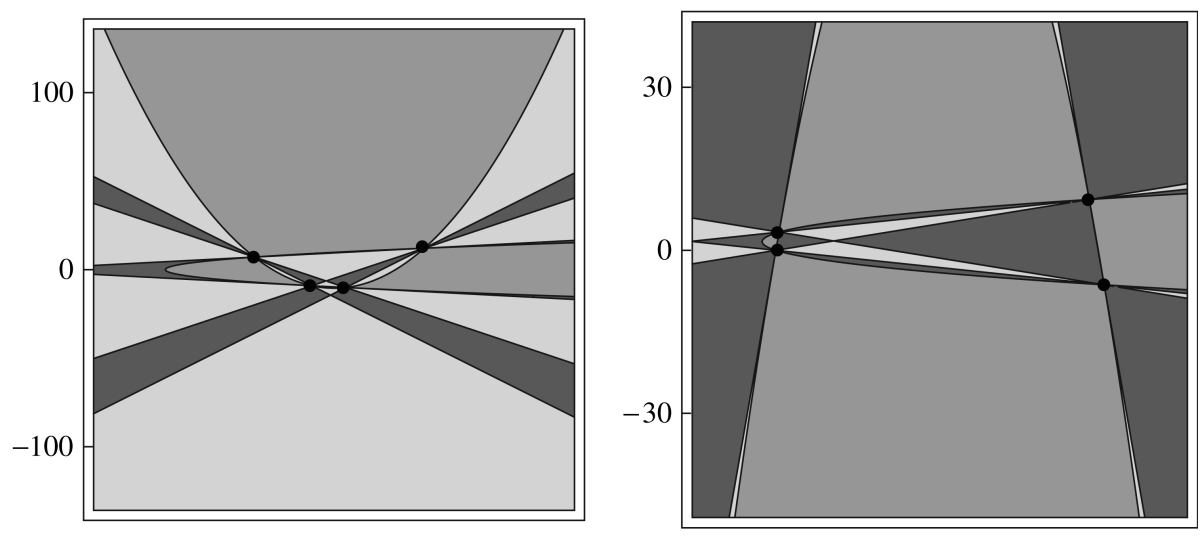

Figure 3. Zones of ellipses and hyperbolas for $I_{10.5}$ in $\left(\tilde{\gamma}_{5}, \delta\right)$ coordinates (left) and $M_{4.35}$ in $\left(\tilde{\gamma}_{6}, \delta\right)$-coordinates (right). The zones are shaded as in Figure 1 .

degenerates into two parallel lines because the quadrilateral is a trapezoid. At these values of $N_{6}$ the direction of the vertical parabola reverses, which is why it is upside down on the right side of Figure 3 .

\section{ProOfS}

7.1. The non-resonant case. Recall from Section 2 that for $n$ non-resonant, the eigenvalues of the Casimir operator $\Omega$ of the projective subalgebra $\mathfrak{a}$ on the composition series $\left\{\mathcal{F}_{n}, \ldots, \mathcal{F}_{n+l-1}\right\}$ of $\mathrm{SQ}_{\lambda, \mu}^{\delta-n, l}$ are distinct. In this case one has the projective quantization, the unique a-equivalence

$$
\mathrm{PQ}_{\lambda, \mu}: \bigoplus_{i=0}^{l-1} \mathcal{F}_{n+i} \rightarrow \mathrm{SQ}_{\lambda, \mu}^{\delta-n, l}
$$

which preserves symbols, in the sense that it maps $\mathcal{F}_{n+i}$ to operators of order $\delta-n-i$, and composing it with the natural symbol map $d x^{\delta} f \partial_{x}^{\delta-n-i} \mapsto d x^{n+i} f$ gives the identity on $\mathcal{F}_{n+i}$.

This map has been the subject of many articles, notably [CMZ97] and [LO99]. It can be used to write the action $L_{\lambda, \mu}$ of $\operatorname{Vect}(\mathbb{R})$ on $\mathrm{SQ}_{\lambda, \mu}^{\delta-n, l}$ in an explicitly $\mathfrak{a}$-diagonal manner:

Definition. Let $\pi^{\lambda, \mu}$ be the representation of $\operatorname{Vect}(\mathbb{R})$ on $\bigoplus_{i=0}^{l-1} \mathcal{F}_{n+i}$ given by

$$
\pi^{\lambda, \mu}(X):=\mathrm{PQ}_{\lambda, \mu}^{-1} \circ\left(\left.L_{\lambda, \mu}(X)\right|_{\mathrm{SQ}_{\lambda, \mu}^{\delta-n, l}}\right) \circ \mathrm{PQ}_{\lambda, \mu} .
$$

Regard $\pi^{\lambda, \mu}$ as an $l \times l$ matrix with entries

$$
\pi_{n+i, n+j}^{\lambda, \mu}: \operatorname{Vect}(\mathbb{R}) \rightarrow \operatorname{Hom}\left(\mathcal{F}_{n+j}, \mathcal{F}_{n+i}\right) .
$$

Recall that if $W$ is any $\operatorname{Vect}(\mathbb{R})$-module, a map $\beta: \operatorname{Vect}(\mathbb{R}) \rightarrow W$ is an a-relative $W$-valued 1 -cochain if it is $\mathfrak{a}$-covariant and zero on $\mathfrak{a}$. The space of all such maps is denoted by $C^{1}(\operatorname{Vect}(\mathbb{R}), \mathfrak{a}, W)$. 
As noted in [LO99], the invariance of the order filtration on $\Psi_{\lambda, \mu}^{\delta-n}$ implies that $\pi^{\lambda, \mu}$ is lower triangular with tensor density actions on the diagonal, and PQ's acovariance forces the subdiagonal entries of $\pi^{\lambda, \mu}$ to be $\mathfrak{a}$-relative $\operatorname{Hom}\left(\mathcal{F}_{n+j}, \mathcal{F}_{n+i}\right)$ valued 1-cochains:

Lemma 7.1. (i) For $i<j, \pi_{n+i, n+j}^{\lambda, \mu}=0$.

(ii) At $j=i, \pi_{n+i, n+i}^{\lambda, \mu}=L_{n+i}$.

(iii) For $i>j, \pi_{n+i, n+j}^{\lambda, \mu} \in C^{1}\left(\operatorname{Vect}(\mathbb{R}), \mathfrak{a}, \operatorname{Hom}\left(\mathcal{F}_{n+j}, \mathcal{F}_{n+i}\right)\right)$.

Our proofs hinge on explicit formulas for the subdiagonal entries $\pi_{n+i, n+j}^{\lambda, \mu}$ for $i-j \leq 4$. These formulas are given in Section 7.8 of [LO99], and they can be deduced for all $i>j$ from the results of [CMZ97]. We now state them in a form similar to but more useful than the form in which they are stated in Co05. The next definition, lemma, and corollary go back essentially to [LO99]; they may be found in roughly the current notation in CS04] and Co05.

Definition. For $\delta \in 2+\mathbb{N}$, let $\beta_{\lambda, \mu}: \operatorname{Vect}(\mathbb{R}) \rightarrow \mathcal{D}_{\lambda, \mu}^{\delta-2}$ be

$$
\beta_{\lambda, \mu}\left(g \partial_{x}\right):=\mathrm{PQ}_{\lambda, \mu}\left(d x^{2} g^{\prime \prime \prime}\right) .
$$

Lemma 7.2. (i) $\beta_{\lambda, \mu}$ is a-relative, and for $r \geq 2$,

$$
\beta_{\lambda, \mu}\left(x^{r+1} \partial_{x}\right):=6(r-2) !^{-1} L_{\lambda, \mu}\left(x^{2} \partial_{x}\right)^{r-2}\left(d x^{\delta} \partial_{x}^{\delta-2}\right) .
$$

(ii) $\operatorname{For} \delta \notin 2+\mathbb{N}, C^{1}\left(\operatorname{Vect}(\mathbb{R}), \mathfrak{a}, \operatorname{Hom}\left(\mathcal{F}_{\lambda}, \mathcal{F}_{\mu}\right)\right)=0$.

(iii) For $\delta \in 2+\mathbb{N}, C^{1}\left(\operatorname{Vect}(\mathbb{R}), \mathfrak{a}, \operatorname{Hom}\left(\mathcal{F}_{\lambda}, \mathcal{F}_{\mu}\right)\right)=\mathbb{C} \beta_{\lambda, \mu}$.

(iv) Under conjugation, $\beta_{\lambda, \mu}^{*}=(-1)^{\delta} \beta_{1-\mu, 1-\lambda}$.

Corollary 7.3.

(i) $\pi_{n+j+1, n+j}^{\lambda, \mu}=0$.

(ii) For $i \geq j+2$, there are scalars $b_{n+i, n+j}(\lambda, \mu)$ such that

$$
\pi_{n+i, n+j}^{\lambda, \mu}=b_{n+i, n+j}(\lambda, \mu) \beta_{n+j, n+i} .
$$

It may be said that these scalars encode the entire $\operatorname{Vect}(\mathbb{R})$-action on $\mathrm{SQ}_{\lambda, \mu}^{\delta-n, l}$ in a maximally efficient manner. In order to give them in the most useful form, we define intermediate scalars $B_{m+r, m}$ for arbitrary $m \in \mathbb{C}$ and $r \in 2+\mathbb{N}$ :

$$
\begin{aligned}
B_{m+r, m}:= & 3^{r / 2}(\lambda+\mu-1-m)_{r} \\
& +3^{r / 2} \frac{2 m-1+r}{r^{2}-1} \sum_{s=0}^{r-2}\left(\begin{array}{c}
r+1 \\
s
\end{array}\right)(2 m-3+r)_{r-s-2}(\lambda+\mu-1-m)_{s} \\
& \times[(r-s+1)(\lambda+\mu-1)-(r-s-1) \delta-(2 m+r+s-1)] .
\end{aligned}
$$

The reader may check that this formula generalizes (2). The point of the common factor $3^{r / 2}$ is to make the expression monic when expressed in terms of $\gamma$.

Theorem 7.4 ([CMZ97] $)$. The scalars $b_{m+r, m}(\lambda, \mu)$ are given by

$$
b_{m+r, m}=\frac{3^{-r / 2}(-1)^{r-1}\left(r^{2}-1\right)(\delta-m)_{r} B_{m+r, m}}{12(2 m-2+2 r)_{r-2}(2 m-1+r)(2 m-3+r)_{r-2}} .
$$


The next proposition gives the parity of the $b_{m+r, m}$ in $\gamma^{1 / 2}$ and in simultaneously reversing the sign of $\delta$ and reflecting across the antidiagonal $m+r=1-m$. It is a manifestation of Lemmas 2.2 and 2.3 and was observed in CMZ97, except for the last sentence, which is obvious by continuity. We remark that these parities are not easy to prove directly.

Proposition 7.5. $\quad$ (i) $\pi_{m+r, m}(1-\mu, 1-\lambda)=(-1)^{r} \pi_{m+r, m}(\lambda, \mu)$.

(ii) Under conjugation, $\pi_{1-m, 1-m-r}(\mu, \lambda)=-\pi_{m+r, m}(\lambda, \mu)^{*}$.

(iii) As functions of $\left(\gamma^{1 / 2}, \delta\right)$, both $b_{m+r, m}$ and $B_{m+r, m}$ are of parity $r$ :

$$
\begin{aligned}
b_{m+r, m}\left(-\gamma^{1 / 2}, \delta\right) & =(-1)^{r} b_{m+r, m}\left(\gamma^{1 / 2}, \delta\right), \\
B_{m+r, m}\left(-\gamma^{1 / 2}, \delta\right) & =(-1)^{r} B_{m+r, m}\left(\gamma^{1 / 2}, \delta\right) .
\end{aligned}
$$

(iv) Under simultaneously reversing the sign of $\delta$ and reflecting across the antidiagonal, $b$ is of parity $r-1$ and $B$ is even:

$$
\begin{aligned}
& b_{1-m, 1-m-r}\left(\gamma^{1 / 2},-\delta\right)=(-1)^{r-1} b_{m+r, m}\left(\gamma^{1 / 2}, \delta\right), \\
& B_{1-m, 1-m-r}\left(\gamma^{1 / 2},-\delta\right)=B_{m+r, m}\left(\gamma^{1 / 2}, \delta\right) .
\end{aligned}
$$

These parities hold for $B_{\bullet}, \bullet$ even when $b_{\bullet}, \bullet$ is undefined.

We shall usually write $B_{m+r, m}$ as a function of $(\gamma, \delta)$, it being understood that in fact it is a function of $\left(\gamma^{1 / 2}, \delta\right)$. We now use Corollary 7.3 to give a general condition under which two non-resonant subquotients are equivalent.

Proposition 7.6. For $l$ arbitrary and n non-resonant with respect to $l, \mathrm{SQ}_{\lambda, \mu}^{\delta-n, l}$ and $\mathrm{SQ}_{\lambda^{\prime}, \mu^{\prime}}^{\delta^{\prime}-n, l}$ are equivalent if and only if there are non-zero scalars $\epsilon_{n}, \epsilon_{n+1}, \ldots, \epsilon_{n+l-1}$ such that for all $(i, j)$ with $0 \leq j \leq i-2$ and $2 \leq i \leq l-1$,

$$
(\delta-n-j)_{i-j} B_{n+i, n+j}(\gamma, \delta) \epsilon_{n+i}=\left(\delta^{\prime}-n-j\right)_{i-j} B_{n+i, n+j}\left(\gamma^{\prime}, \delta^{\prime}\right) \epsilon_{n+j} .
$$

Proof. The two subquotients are equivalent if and only if the corresponding representations $\pi^{\lambda, \mu}$ and $\pi^{\lambda^{\prime}, \mu^{\prime}}$ on $\bigoplus_{i=0}^{l-1} \mathcal{F}_{n+i}$ are equivalent. Note that both $\pi^{\lambda, \mu}$ and $\pi^{\lambda^{\prime}, \mu^{\prime}}$ carry the Casimir operator $\Omega$ to the same block-diagonal matrix with scalars on the diagonal:

$$
\pi_{n+i, n+j}^{\lambda, \mu}(\Omega)=\pi_{n+i, n+j}^{\lambda^{\prime}, \mu^{\prime}}(\Omega)=\delta_{i, j}(n+i)(n+i-1),
$$

where $\delta_{i, j}$ is the Kronecker delta function. Since $n$ is non-resonant, these scalars are distinct.

Suppose that $\epsilon$ is an endomorphism of $\bigoplus_{i=0}^{l-1} \mathcal{F}_{n+i}$ intertwining $\pi^{\lambda, \mu}$ and $\pi^{\lambda^{\prime}, \mu^{\prime}}$. Since it commutes with the $\Omega$ action, it must be block-diagonal: write $\epsilon_{n}, \ldots, \epsilon_{n+l-1}$ for its diagonal entries. Since the diagonal entries $\pi_{n+i, n+i}^{\lambda, \mu}$ and $\pi_{n+i, n+i}^{\lambda^{\prime}, \mu^{\prime}}$ are both $L_{n+i}, \epsilon_{n+i}$ must intertwine $L_{n+i}$ with itself. It is elementary that this forces $\epsilon_{n+i}$ to be a scalar. By Corollary 7.3 and Theorem [7.4, (11) is the condition for $\epsilon$ to intertwine $\pi^{\lambda, \mu}$ with $\pi^{\lambda^{\prime}, \mu^{\prime}}$.

Proofs of Propositions 3.1, 3.2, 3.3, and 6.1 and Theorem 3.4. These are all essentially corollaries of Proposition [7.6, and we will only discuss Theorem 3.4 and Proposition 6.1. In Theorem 3.4, it is obvious from (11) that the SVC is necessary for equivalence. Assuming that it holds, (11) gives one condition on the ratios of the scalars $\epsilon_{n+i}$ for each of the six quantities (6) that is non-zero. Since there are five scalars and hence four independent ratios in length 5 , there are in general 
two additional conditions on $(\gamma, \delta)$ which must be satisfied in order for (11) to be soluble.

For example, suppose that none of the quantities (6) vanish. Rewrite (11):

$$
\frac{\epsilon_{n+i}}{\epsilon_{n+j}}=\frac{\left(\delta^{\prime}-n-j\right)_{i-j}}{(\delta-n-j)_{i-j}} \frac{B_{n+i, n+j}\left(\gamma^{\prime}, \delta^{\prime}\right)}{B_{n+i, n+j}(\gamma, \delta)} .
$$

Solving these equations for $(i, j)$ equal to $(2,0),(3,1),(4,2)$, and $(3,0)$ determines the $\epsilon_{n+i}$ up to a common multiplicative scalar. The reader may easily check that they are soluble at the two remaining places $(4,1)$ and $(4,0)$ if and only if Part (v) of Theorem 3.4 holds.

For Proposition 6.1, write $x \cdot B$ and $y \cdot B$ for the numerator and denominator of the displayed equation. It is easy to check that

$$
\frac{x \cdot B\left(\gamma^{\prime}, \delta^{\prime}\right)}{x \cdot B(\gamma, \delta)}=\frac{(\delta-n)_{4}(\delta-n-1)_{2}}{\left(\delta^{\prime}-n\right)_{4}\left(\delta^{\prime}-n-1\right)_{2}} \frac{\epsilon_{n+4} \epsilon_{n+3}}{\epsilon_{n+1} \epsilon_{n}},
$$

independent of $x$. The invariance follows, and we leave the reader to check the completeness of $\tilde{I}_{n}$ and $\tilde{J}_{n}$.

7.2. The resonant case. Recall from Section 2 that $n$ is resonant with respect to $l$ if $n \in-\frac{1}{2} \mathbb{N}$ and $n+l-1 \geq 1$. In this case some of the eigenvalues of $\Omega$ on the composition series $\left\{\mathcal{F}_{n}, \ldots, \mathcal{F}_{n+l-1}\right\}$ of $\mathrm{SQ}_{\lambda, \mu}^{\delta-n, l}$ are doubled: those for which $n+i$ and $n+j$ add to 1 .

Here there is in general no a-equivalence of the form (8), but it is possible to choose an affine quantization $\overline{\mathrm{PQ}}_{\lambda, \mu}$ in place of $\mathrm{PQ}_{\lambda, \mu}$ which is as close as possible to a projective quantization, in the sense that it preserves the generalized eigenspaces of $\Omega$. By an affine quantization we mean a symbol-preserving affine equivalence, where the affine algebra $\mathfrak{b}$ is the Borel subalgebra of $\mathfrak{a}$ defined by

$$
\mathfrak{b}:=\operatorname{Span}_{\mathbb{C}}\left\{\partial_{x}, x \partial_{x}\right\} .
$$

Our derivation of the resonant equivalence classes relies on the explicit form of the representation $\bar{\pi}^{\lambda, \mu}$ taking the place of $\pi^{\lambda, \mu}$ in (9). We now recall from CS04 the formulas for the matrix entries $\bar{\pi}_{n+i, n+j}^{\lambda, \mu}$. In fact we will give more detail than is needed for the current article, because we take this opportunity to give in Theorem 7.10 a significant simplification of the formulas for the scalars $\bar{b}_{n+i, n+j}(\lambda, \mu)$ given in Theorem 6.3 of [CS04]. We begin with the definitions and properties of the two new types of 1-cochains occurring in the entries of $\bar{\pi}^{\lambda, \mu}$.

Write $\partial$ for the coboundary operator: if $T \in \mathcal{D}_{\lambda, \mu}$ is 0 -cochain and $\omega: \operatorname{Vect}(\mathbb{R}) \rightarrow$ $\mathcal{D}_{\lambda, \mu}$ is a 1-cochain, then their coboundaries are, respectively, the 1- and 2-cochains

$$
\partial T(X)=L_{\lambda, \mu}(X) T, \quad \partial \omega(X \wedge Y)=L_{\lambda, \mu}(X) \omega(Y)-L_{\lambda, \mu}(Y) \omega(X)-\omega([X, Y]) .
$$

A 2-cochain $\theta: \Lambda^{2} \operatorname{Vect}(\mathbb{R}) \rightarrow \mathcal{D}_{\lambda, \mu}$ is said to be a-relative if it is a-covariant and zero on $\mathfrak{a} \wedge \operatorname{Vect}(\mathbb{R})$.

Consider the following three properties a 1-cochain $\omega$ may have:
(i) $\omega$ is zero on $\mathfrak{a}$,
(ii) $\omega$ is a-covariant,
(iii) $\partial \omega$ is $\mathfrak{a}$-relative.

If $\omega$ has any two of these properties, then it has the third and is a-relative. However, it is possible to have any one of these properties but neither of the other two.

We will also need the cup product of a $\mathcal{D}_{\nu, \mu}$-valued 1-cochain $\omega_{\nu, \mu}$ with a $\mathcal{D}_{\lambda, \nu^{-}}$ valued 1-cochain $\omega_{\lambda, \nu}$ :

$$
\omega_{\nu, \mu} \cup \omega_{\lambda, \nu}(X \wedge Y):=\omega_{\nu, \mu}(X) \circ \omega_{\lambda, \nu}(Y)-\omega_{\nu, \mu}(Y) \circ \omega_{\lambda, \nu}(X) .
$$


Definition. For $\delta \in 1+\mathbb{N}$, let $\alpha_{\lambda, \mu}: \operatorname{Vect}(\mathbb{R}) \rightarrow \mathcal{D}_{\lambda, \mu}$ be

$$
\alpha_{\lambda, \mu}:=\frac{2 \sqrt{3}}{\delta \gamma^{1 / 2}}\left(\frac{1}{12} \delta(\delta-1)(\delta+1-\gamma) \beta_{\lambda, \mu}-\partial\left(d x^{\delta} \partial_{x}^{\delta}\right)\right) .
$$

Note that although $\beta_{\lambda, \mu}$ is not defined at $\delta=1$, it does not appear in the formula there. For $\delta \in 2+\mathbb{N}, \alpha_{\lambda, \mu}$ is manifestly cohomologous to a multiple of $\beta_{\lambda, \mu}$ except in the self-adjoint case $\gamma=0$, where it appears to be undefined. It is a crucial point in CS04 that in fact the formula for $\alpha_{\lambda, \mu}$ has a removable singularity at $\gamma=0$, because this is exactly where it is needed. At this value, $\beta_{\lambda, \mu}$ is a coboundary and the restriction $\left.\alpha_{\lambda, \mu}\right|_{\mathfrak{a}}$ is a non-trivial cocycle.

We define the lacunary differential operator modules in terms of the lacunary pseudodifferential operator modules of Section 5 , for $k \in \mathbb{N}$ and $k \neq \delta$,

$$
\mathcal{D}_{\lambda, \mu}^{k, \text { lac }}:=\mathcal{D}_{\lambda, \mu} \cap \Psi_{\lambda, \mu}^{k, \text { lac }} .
$$

Lemma 7.7. (i) $\alpha_{\lambda, \mu}$ is $\mathfrak{b}$-relative, $\mathcal{D}_{\lambda, \mu}^{\delta-1, \text { lac }}$-valued, and has symbol

$$
\alpha_{\lambda, \mu}\left(g \partial_{x}\right) \equiv d x^{\delta} g^{\prime \prime} \partial_{x}^{\delta-1} .
$$

(ii) For $\delta \notin 1+\mathbb{N}, C^{1}\left(\operatorname{Vect}(\mathbb{R}), \mathfrak{b}, \operatorname{Hom}\left(\mathcal{F}_{\lambda}, \mathcal{F}_{\mu}\right)\right)=0$.

(iii) For $\delta=1, C^{1}\left(\operatorname{Vect}(\mathbb{R}), \mathfrak{b}, \operatorname{Hom}\left(\mathcal{F}_{\lambda}, \mathcal{F}_{\mu}\right)\right)=\mathbb{C} \alpha_{\lambda, \mu}$ and $\partial \alpha_{\lambda, \mu}=0$.

(iv) For $\delta \in 2+\mathbb{N}, \partial \alpha_{\lambda, \mu}$ is a-relative. Moreover,

$$
\left\{\omega \in C^{1}\left(\operatorname{Vect}(\mathbb{R}), \mathfrak{b}, \mathcal{D}_{\lambda, \mu}^{\delta-1, \text { lac }}\right): \partial \omega \text { is a-relative }\right\}=\mathbb{C} \alpha_{\lambda, \mu} \text {, and }
$$

$\left\{\omega \in C^{1}\left(\operatorname{Vect}(\mathbb{R}), \mathfrak{b}, \operatorname{Hom}\left(\mathcal{F}_{\lambda}, \mathcal{F}_{\mu}\right)\right): \partial \omega\right.$ is a-relative $\}=\operatorname{Span}_{\mathbb{C}}\left\{\alpha_{\lambda, \mu}, \beta_{\lambda, \mu}\right\}$.

(v) Under conjugation, $\alpha_{\lambda, \mu}^{*}=(-1)^{\delta-1} \alpha_{1-\mu, 1-\lambda}$.

Thus $\alpha_{\lambda, \mu}$ is $\mathfrak{b}$-relative with $\mathfrak{a}$-relative coboundary, but is not zero on $\mathfrak{a}$. In the self-adjoint case it is known to be a cocycle if and only if $1 \leq \delta \leq 4$. Let us remark that $\alpha_{\lambda, \mu}\left(g \partial_{x}\right)$ is in general not $\mathrm{PQ}_{\lambda, \mu}\left(d x g^{\prime \prime}\right)$ : a formula for the difference is given in Proposition 9.2 of [CS04.

The second new type of cochain is zero on $\mathfrak{a}$ but does not have an $\mathfrak{a}$-relative coboundary. It has two variations:

Definition. For $\nu-\lambda \in 1+\mathbb{N}$ and $\mu-\nu \in 2+\mathbb{N}$, let $\Delta_{\lambda, \mu}^{\nu, \text { lower }}: \operatorname{Vect}(\mathbb{R}) \rightarrow \mathcal{D}_{\lambda, \mu}$ be

$$
\Delta_{\lambda, \mu}^{\nu, \text { lower }}:=-2\left(\beta_{\nu, \mu} \circ\left(d x^{\nu-\lambda} \partial_{x}^{\nu-\lambda}\right)-\beta_{\lambda, \mu}\right) /(\nu-\lambda)(\nu+\lambda-1) .
$$

For $\nu-\lambda \in 2+\mathbb{N}$ and $\mu-\nu \in 1+\mathbb{N}$, let $\Delta_{\lambda, \mu}^{\nu \text {,upper }}: \operatorname{Vect}(\mathbb{R}) \rightarrow \mathcal{D}_{\lambda, \mu}$ be

$$
\Delta_{\lambda, \mu}^{\nu, \text { upper }}:=2\left(\left(d x^{\mu-\nu} \partial_{x}^{\mu-\nu}\right) \circ \beta_{\lambda, \nu}-\beta_{\lambda, \mu}\right) /(\mu-\nu)(\mu+\nu-1) .
$$

Just as for $\alpha_{\lambda, \mu}$, it is a key point in CS04 that $\Delta_{\lambda, \mu}^{\nu, \text { lower }}$ and $\Delta_{\lambda, \mu}^{\nu \text {,upper }}$ have removable singularities at $\nu+\lambda=1$ and $\mu+\nu=1$, so we may consider them to be defined at such values. In that article, $\Delta_{\lambda, \mu}^{\nu, \text { lower }}$ is called $\delta_{\mu-\lambda, \nu-\lambda}(\lambda)$ and $\Delta_{\lambda, \mu}^{\nu, \text { upper }}$ is called $\delta_{\mu-\nu, \mu-\lambda}(\lambda)$. The superscripts "lower" and "upper" refer to the location of these 1-cochains among the matrix entries of $\bar{\pi}^{\lambda, \mu}$ : as explained in CS04, they occur in the lower singular triangle and upper singular triangle, respectively.

Lemma 7.8. $\quad$ (i) $\Delta_{\lambda, \mu}^{\nu, \text { lower }}$ and $\Delta_{\lambda, \mu}^{\nu \text {,upper }}$ are $\mathfrak{b}$-relative, $\mathcal{D}_{\lambda, \mu}^{\delta-3}$-valued, and have symbols

$$
-\Delta_{\lambda, \mu}^{\nu, \text { lower }}\left(g \partial_{x}\right) \equiv \Delta_{\lambda, \mu}^{\nu, \text { upper }}\left(g \partial_{x}\right) \equiv \frac{1}{2} d x^{\delta} g^{\prime \prime \prime \prime} \partial_{x}^{\delta-3} .
$$


(ii) $\Delta_{\lambda, \mu}^{\nu, \text { lower }}$ is the unique $\mathcal{D}_{\lambda, \mu}^{\delta-3}$-valued 1-cochain such that the following 2cochain is a-relative:

$$
\partial \Delta_{\lambda, \mu}^{\nu, \text { lower }}+\beta_{\nu, \mu} \cup \alpha_{\lambda, \nu}+2\left(\partial \beta_{\nu, \mu}\right) \circ\left(d x^{\nu-\lambda} \partial_{x}^{\nu-\lambda}\right) /(\nu-\lambda)(\nu+\lambda-1) .
$$

(iii) $\Delta_{\lambda, \mu}^{\nu \text {,upper }}$ is the unique $\mathcal{D}_{\lambda, \mu}^{\delta-3}$-valued 1-cochain such that the following 2 cochain is a-relative:

$$
\partial \Delta_{\lambda, \mu}^{\nu, \text { upper }}+\alpha_{\nu, \mu} \cup \beta_{\lambda, \nu}+2\left(d x^{\mu-\nu} \partial_{x}^{\mu-\nu}\right) \circ\left(\partial \beta_{\lambda, \nu}\right) /(\mu-\nu)(\mu+\nu-1) .
$$

(iv) Conjugation exchanges $\Delta_{\lambda, \mu}^{\nu, \text { lower }}$ and $(-1)^{\delta} \Delta_{1-\mu, 1-\lambda}^{1-\nu, \text { upper }}$.

Remark. Parts (ii) and (iii) of this lemma correct an error in Lemma 6.2 of [CS04, where the summands containing $\partial \beta_{\nu, \mu}$ and $\partial \beta_{\lambda, \nu}$ were omitted. This error does not affect the other results of [CS04].

Suppose now that $n$ is resonant. Then it is not hard to prove that there exists a $\mathfrak{b}$-equivalence

$$
\overline{\mathrm{PQ}}_{\lambda, \mu}: \bigoplus_{i=0}^{l-1} \mathcal{F}_{n+i} \rightarrow \mathrm{SQ}_{\lambda, \mu}^{\delta-n, l}
$$

which preserves symbols and also preserves the generalized eigenspaces of $\Omega$ : for all $0 \leq i<j \leq l-1$ such that $2 n+i+j=1, \overline{\mathrm{PQ}}_{\lambda, \mu}$ maps $\mathcal{F}_{n+i} \oplus \mathcal{F}_{n+j}$ to the $(n+i)(n+i-1)$-generalized eigenspace of $\Omega$ on $\mathrm{SQ}_{\lambda, \mu}^{\delta-n, l}$.

Such equivalences are not unique. Given one, define a representation $\bar{\pi}^{\lambda, \mu}$ of $\operatorname{Vect}(\mathbb{R})$ on $\bigoplus_{i=0}^{l-1} \mathcal{F}_{n+i}$ by

$$
\bar{\pi}^{\lambda, \mu}(X):=\overline{\mathrm{PQ}}_{\lambda, \mu}^{-1} \circ\left(\left.L_{\lambda, \mu}(X)\right|_{\mathrm{SQ}_{\lambda, \mu}^{\delta-n, l}}\right) \circ \overline{\mathrm{PQ}}_{\lambda, \mu} .
$$

Regard $\bar{\pi}^{\lambda, \mu}$ as an $l \times l$ matrix with entries

$$
\bar{\pi}_{n+i, n+j}^{\lambda, \mu}: \operatorname{Vect}(\mathbb{R}) \rightarrow \operatorname{Hom}\left(\mathcal{F}_{n+j}, \mathcal{F}_{n+i}\right) .
$$

Then just as in Lemma 7.1, $\bar{\pi}^{\lambda, \mu}$ is lower triangular with tensor density actions on the diagonal: for $i<j, \bar{\pi}_{n+i, n+j}^{\lambda, \mu}=0$, and at $j=i, \bar{\pi}_{n+i, n+i}^{\lambda, \mu}=L_{n+i}$.

We are now in position to state the resonant analogs of Corollary 7.3 and Theorem 7.4. Parts (i)-(iii) of Proposition 7.9 and Parts (i) and (ii) of Theorem 7.10 were proven in Ga00, and the remaining parts of the two statements comprise the main results of CS04. In fact, as we mentioned, Parts (iii) and (iv) of Theorem 7.10 significantly simplify the formulas obtained in [CS04, and we will give a proof of this simplification.

Proposition 7.9. $\overline{\mathrm{PQ}}_{\lambda, \mu}$ may be chosen so that $\bar{\pi}^{\lambda, \mu}$ has the properties listed below. In the integral resonant case there is a 1-parameter family of such choices, and in the half-integral resonant case there is only one.

(i) For $i=j+1$ and $n+j \neq 0, \bar{\pi}_{n+j+1, n+j}^{\lambda, \mu}=0$.

(ii) For $i \geq j+2, \bar{\pi}_{n+i, n+j}^{\lambda, \mu}$ is given by (10) in all of the following cases:

$$
n+j \geq \frac{1}{2}, \quad n+i \leq \frac{1}{2}, \quad 2 n+i+j=0, \quad 2 n+i+j=2 .
$$

(iii) For $i>j$ and $n+i=1-n-j$, there are scalars $a_{1-n-j, n+j}(\lambda, \mu)$ such that

$$
\bar{\pi}_{1-n-j, n+j}^{\lambda, \mu}=a_{1-n-j, n+j}(\lambda, \mu) \alpha_{n+j, 1-n-j} .
$$


(iv) For $n+j \leq 0$ and $2 n+i+j \geq 3$, there are scalars $\bar{b}_{n+i, n+j}(\lambda, \mu)$ such that

$$
\begin{aligned}
\bar{\pi}_{n+i, n+j}^{\lambda, \mu}= & \bar{b}_{n+i, n+j}(\lambda, \mu) \beta_{n+j, n+i} \\
& +b_{n+i, 1-n-j}(\lambda, \mu) a_{1-n-j, n+j}(\lambda, \mu) \Delta_{n+j, n+i}^{1-n-j, \text { lower }} .
\end{aligned}
$$

(v) For $n+i \geq 1$ and $2 n+i+j \leq-1$, there are scalars $\bar{b}_{n+i, n+j}(\lambda, \mu)$ such that

$$
\begin{aligned}
\bar{\pi}_{n+i, n+j}^{\lambda, \mu}= & \bar{b}_{n+i, n+j}(\lambda, \mu) \beta_{n+j, n+i} \\
& +a_{n+i, 1-n-i}(\lambda, \mu) b_{1-n-i, n+j}(\lambda, \mu) \Delta_{n+j, n+i}^{1-n-i, \text { upper }} .
\end{aligned}
$$

Theorem 7.10. $\quad$ (i) The scalar $a_{1,0}(\lambda, \mu)$ is $-\delta \gamma^{1 / 2} / 2 \sqrt{3}$.

(ii) For $m \in-\frac{1}{2} \mathbb{Z}^{+}$, the scalars $a_{1-m, m}(\lambda, \mu)$ are given by

$a_{1-m, m}=-\frac{(\delta-m)_{1-2 m} B_{1-m, m}}{2 \cdot 3^{(1-2 m) / 2}(-2 m) !^{2}}=-\frac{(\delta-m)_{1-2 m}(\lambda+\mu-1-m)_{1-2 m}}{2(-2 m) !^{2}}$.

(iii) For $m \in-\frac{1}{2} \mathbb{Z}^{+}$and $2 m+r \geq 3$, the scalars $\bar{b}_{m+r, m}(\lambda, \mu)$ are given by

$$
\begin{aligned}
& \bar{b}_{m+r, m}= \frac{3^{-r / 2}(-1)^{2 m-1+r}\left(r^{2}-1\right)(\delta-m)_{r}}{12(2 m-2+2 r)_{r-2}(2 m-1+r)(2 m-3+r) !(-2 m) !} \\
& \times\left[\left.\frac{1}{2} \frac{\partial}{\partial u}\right|_{u=m}\left(B_{u+r, u}-B_{u+r, u+1-2 m} B_{u+1-2 m, u}\right)\right. \\
& \\
&\left.\quad-\left(\frac{1}{1-2 m}+\frac{2 r}{r^{2}-1}-\frac{2(2 m-1+r)}{(2 m-1+r)^{2}-1}\right) B_{m+r, 1-m} B_{1-m, m}\right] .
\end{aligned}
$$

(iv) For $r \geq 3$, the scalars $\bar{b}_{r, 0}(\lambda, \mu)$ are given by

$$
\begin{aligned}
\bar{b}_{r, 0}=\frac{3^{-r / 2}(-1)^{r-1}(\delta)_{r}}{12(2 r-2)_{r-3}(r-3) !} & {\left[\left.\frac{1}{2} \frac{\partial}{\partial u}\right|_{u=0}\left(B_{u+r, u}-\gamma^{1 / 2} B_{u+r, u+1}\right)\right.} \\
& \left.-\left(1+\frac{2 r}{r^{2}-1}-\frac{2(r-1)}{r^{2}-2 r}\right) \gamma^{1 / 2} B_{r, 1}\right] .
\end{aligned}
$$

All of the scalars $B_{\bullet}, \bullet$ in this theorem are evaluated at $(\gamma, \delta)$.

Proof. Parts (i) and (ii) are proven in both Ga00] and [CS04. Parts (iii) and (iv) are proven in CS04, except that more complicated formulas for $\bar{b}_{m+r, m}$ are given. After a long and delicate but elementary computation starting from the CS04] formulas, one arrives at the formulas above, except that instead of the partial derivative given in Part (iii) one has

$$
\left.\lim _{\epsilon \rightarrow 0} \epsilon^{-1}\left(B_{m+\epsilon+r, m+\epsilon}-B_{m+\epsilon+r, 1-m+\epsilon} B_{1-m+\epsilon, m+\epsilon}\right)\right|_{\lambda, \mu+\epsilon},
$$

and instead of the partial derivative given in Part (iv) one has

$$
\left.\lim _{\epsilon \rightarrow 0} \epsilon^{-1}\left(B_{\epsilon+r, \epsilon}-\gamma^{1 / 2} B_{\epsilon+r, 1+\epsilon}\right)\right|_{\lambda, \mu+\epsilon},
$$

Here evaluation at $(\lambda, \mu+\epsilon)$ means that instead of using the usual values $3(\lambda+\mu-1)^{2}$ and $\mu-\lambda$ for $\gamma$ and $\delta$, we use $3(\lambda+\mu-1+\epsilon)^{2}$ and $\mu-\lambda+\epsilon$.

By [CS04], these limits exist. Therefore

$$
B_{m+r, m}=B_{m+r, 1-m} B_{1-m, m} \text { and } B_{r, 0}=\gamma^{1 / 2} B_{r, 1}
$$


for all $(\gamma, \delta)$. This is not obvious directly from the formula for $B_{\bullet}, \bullet$ and is stated below in Corollary 7.12

Consequently, the two limits displayed above are in fact directional derivatives along the vector $(1,0,1)$ of

$$
B_{u+r, u}-B_{u+r, u+1-2 m} B_{u+1-2 m, u} \text { and } B_{u+r, u}-\gamma^{1 / 2} B_{u+r, u+1},
$$

regarded as functions of $(u, \lambda, \mu)$. For the first function the derivative is evaluated at $(m, \lambda, \mu)$, and for the second it is evaluated at $(0, \lambda, \mu)$. By Corollary [7.12, at these values of $u$ the two functions are identically zero for all values of $\lambda$ and $\mu$, so the result is unchanged if we take the directional derivative along $(1,0,0)$ instead. The result follows.

Note that the formula for the scalars $\bar{b}$ occurring in Part (v) of Proposition 7.9 is not included in Theorem 7.10. This is because it may be deduced from Parts (iii) and (iv) of Theorem 7.10 together with the following analog of Proposition [7.5, which is given as Proposition 9.1 in [CS04].

Proposition 7.11. (i) The parity equations satisfied by the matrices $\pi$ in Parts (i) and (ii) of Proposition 7.5 are also satisfied by the matrices $\bar{\pi}$.

(ii) The parity equations satisfied by the scalars $b$ in Parts (iii) and (iv) of Proposition 7.5 are also satisfied by the scalars $\bar{b}$. In particular, for $m+r \in$ $1+\frac{1}{2} \mathbb{N}$ and $2 m+r \leq-1$ we have

$$
\bar{b}_{m+r, m}\left(\gamma^{1 / 2}, \delta\right)=(-1)^{r-1} \bar{b}_{1-m, 1-m-r}\left(\gamma^{1 / 2},-\delta\right) .
$$

(iii) The scalars a satisfy the following parity equations:

$$
a_{1-m, m}\left(-\gamma^{1 / 2}, \delta\right)=(-1)^{1-2 m} a_{1-m, m}\left(\gamma^{1 / 2}, \delta\right)=a_{1-m, m}\left(\gamma^{1 / 2},-\delta\right) .
$$

These results have the following corollary, which is not noticed in CS04]. Half of it was already proven in the proof of Theorem 7.10, the other half follows from Part (iv) of Proposition [7.5. The simplest examples, $B_{3,0}$ and $B_{1,-2}$, may be observed in the formulas displayed below Proposition 4.1 .

Corollary 7.12.

(i) For $m \in-\frac{1}{2} \mathbb{Z}^{+}$and $2 m+r \geq 3$,

$$
B_{m+r, m}=B_{m+r, 1-m} B_{1-m, m} \text {. }
$$

(ii) For $m+r \in 1+\frac{1}{2} \mathbb{Z}^{+}$and $2 m+r \leq-1$,

$$
B_{m+r, m}=B_{m+r, 1-m-r} B_{1-m-r, m} .
$$

(iii) For $r \geq 3, B_{r, 0}=\gamma^{1 / 2} B_{r, 1}$ and $B_{1,1-r}=\gamma^{1 / 2} B_{0,1-r}$.

We have now simplified the results of [CS04] sufficiently to give a useful criterion for equivalence of resonant subquotients, analogous to Proposition [7.6 We first state a lemma we will need; its proof is an easy exercise.

Lemma 7.13. $\operatorname{Hom}_{\mathfrak{b}}\left(\mathcal{F}_{\lambda}, \mathcal{F}_{\mu}\right)$ is $\mathbb{C} d x^{\delta} \partial_{x}^{\delta}$ if $\delta \in \mathbb{N}$, and 0 otherwise.

Proposition 7.14. For $n$ resonant with respect to $l, \mathrm{SQ}_{\lambda, \mu}^{\delta-n, l}$ and $\mathrm{SQ}_{\lambda^{\prime}, \mu^{\prime}}^{\delta^{\prime}-n, l}$ are equivalent if and only if there are non-zero scalars $\epsilon_{n}, \epsilon_{n+1}, \ldots, \epsilon_{n+l-1}$ and an arbitrary scalar $\zeta$ such that

(i) For $i \geq j+2$, (11) holds in all of the following cases:

$$
n+j \geq \frac{1}{2}, \quad n+i \leq \frac{1}{2}, \quad \text { or } \quad 2 n+i+j \in\{0,1,2\} .
$$


(ii) If $n$ is integral, then $\delta \gamma^{1 / 2} \epsilon_{1}=\delta^{\prime} \gamma^{1 / 2} \epsilon_{0}$.

(iii) For $n+j \leq-\frac{1}{2}$ and $2 n+i+j \geq 3$ or $n+i \geq \frac{3}{2}$ and $2 n+i+j \leq-1$,

$$
\bar{b}_{n+i, n+j}(\gamma, \delta) \epsilon_{n+i}=\bar{b}_{n+i, n+j}\left(\gamma^{\prime}, \delta^{\prime}\right) \epsilon_{n+j} .
$$

For $n+j \leq-\frac{1}{2}$ and $2 n+i+j \geq 3$, this condition is equivalent to

$$
\begin{aligned}
& \left.\epsilon_{n+i}(\delta-n-j)_{i-j} \frac{\partial}{\partial u}\right|_{u=n+j}\left(B_{u+r, u}-B_{u+r, u+1-2 m} B_{u+1-2 m, u}\right) \\
= & \left.\epsilon_{n+j}\left(\delta^{\prime}-n-j\right)_{i-j} \frac{\partial}{\partial u}\right|_{u=n+j}\left(B_{u+r, u}^{\prime}-B_{u+r, u+1-2 m}^{\prime} B_{u+1-2 m, u}^{\prime}\right),
\end{aligned}
$$

where $B_{\bullet, \bullet}$ is evaluated at $(\gamma, \delta)$ and $B_{\bullet, \bullet}^{\prime}$ denotes evaluation at $\left(\gamma^{\prime}, \delta^{\prime}\right)$.

(iv) For $n$ integral, $n+j=0$, and $n+i \geq 3$,

$$
\bar{b}_{n+i, 0}(\gamma, \delta) \epsilon_{n+i}=\bar{b}_{n+i, 0}\left(\gamma^{\prime}, \delta^{\prime}\right) \epsilon_{0}+b_{n+i, 1}\left(\gamma^{\prime}, \delta^{\prime}\right) \zeta .
$$

(v) For $n$ integral, $n+i=1$, and $n+j \leq-2$,

$$
\bar{b}_{1, n+j}(\gamma, \delta) \epsilon_{1}+b_{0, n+j}(\gamma, \delta) \zeta=\bar{b}_{1, n+j}\left(\gamma^{\prime}, \delta^{\prime}\right) \epsilon_{n+j} .
$$

Proof. We follow the approach taken in proving Proposition 7.6. keeping in mind the formulas of Proposition 7.9 and Theorem 7.10, The two subquotients are equivalent if and only if the representations $\bar{\pi}^{\lambda, \mu}$ and $\bar{\pi}^{\lambda^{\prime}, \mu^{\prime}}$ are equivalent. Suppose that $\epsilon$ is an endomorphism of $\bigoplus_{i=0}^{l-1} \mathcal{F}_{n+i}$ intertwining $\bar{\pi}^{\lambda, \mu}$ and $\bar{\pi}^{\lambda^{\prime}, \mu^{\prime}}$. Regard it as a block matrix with entries $\epsilon_{n+i, n+j}: \mathcal{F}_{n+i} \rightarrow \mathcal{F}_{n+j}$ as usual. The fact that $\epsilon$ preserves the generalized eigenspaces of $\Omega$ implies that $\epsilon_{n+i, n+j}=0$, except possibly on the diagonal $i=j$ and the antidiagonal $2 n+i+j=1$.

Let $\epsilon_{n}, \ldots, \epsilon_{n+l-1}$ be the diagonal entries of $\epsilon$. Recall that the diagonal entries of the two representations are $\bar{\pi}_{m, m}^{\lambda, \mu}=\bar{\pi}_{m, m}^{\lambda^{\prime}, \mu^{\prime}}=L_{m}$ and that these representations both arise from affine quantizations, so their restrictions to $\mathfrak{b}$ are diagonal. Therefore by Lemma 7.13 the $\epsilon_{m}$ are scalars, and the antidiagonal entries $\epsilon_{1-m, m}$ are multiples of the Bol operators

$$
\mathrm{Bol}_{m, 1-m}:=d x^{1-2 m} \partial_{x}^{1-2 m}: \mathcal{F}_{m} \rightarrow \mathcal{F}_{1-m},
$$

which are known to be a-maps. Observe that $\mathrm{Bol}_{0,1}$ is the de Rham differential $d$, a $\operatorname{Vect}(\mathbb{R})$-map.

Consider the intertwining equation $\epsilon \circ \bar{\pi}^{\lambda, \mu}=\bar{\pi}^{\lambda^{\prime}, \mu^{\prime}} \circ \epsilon$. For $n+j \geq \frac{1}{2}$ or $n+i \leq \frac{1}{2}$, its $(n+i, n+j)$ entry is $\bar{\pi}_{n+i, n+j}^{\lambda, \mu} \epsilon_{n+i}=\bar{\pi}_{n+i, n+j}^{\lambda^{\prime}, \mu^{\prime}} \epsilon_{n+j}$, which yields Part (i) in these cases.

For $2 n+i+j=2$, the $(n+i, n+j)$ entry of the intertwining equation is

$$
\bar{\pi}_{2-n-i, n+i}^{\lambda, \mu} \epsilon_{n+i}=\bar{\pi}_{2-n-i, n+i}^{\lambda^{\prime}, \mu^{\prime}} \epsilon_{n+i}+\bar{\pi}_{2-n-i, 1-n-i}^{\lambda^{\prime}, \mu^{\prime}} \epsilon_{1-n-i, n+i} .
$$

Since $\bar{\pi}_{m+1, m}=0$ for $(m+1, m) \neq(1,0)$, we obtain Part (i) in this case also. The proof of Part (i) for $2 n+i+j=0$ is similar.

On the antidiagonal $2 n+i+j=1$, recall that the $(1-m, m)$-entry $\bar{\pi}_{1-m, m}^{\lambda, \mu}$ is $a_{1-m, m} \alpha_{1-m, m}$. Use the corresponding entry of the intertwining equation to obtain the proportionality

$$
\left(\epsilon_{1-m} a_{1-m, m}(\lambda, \mu)-\epsilon_{m} a_{1-m, m}\left(\lambda^{\prime}, \mu^{\prime}\right)\right) \alpha_{1-m, m}=\partial \epsilon_{1-m, m} \propto \partial \operatorname{Bol}_{m, 1-m} .
$$

It is proven in CS04 that $\partial \mathrm{Bol}_{m, 1-m}=\frac{1}{12}(2-2 m)_{2} \beta_{m, 1-m}$, a non-zero multiple of $\beta_{1-m, m}$ unless $m=0$. (Indeed, this is why $\alpha_{\lambda, \mu}$ is defined at $\lambda+\mu=1$.) Since 
$\alpha_{1-m, m}$ and $\beta_{1-m, m}$ are linearly independent, both sides of the above displayed equation must be zero. This completes the proof of Part (i) and also proves Part (ii).

The argument in the last paragraph also gives $\epsilon_{1-m, m}=0$ for $m \neq 0$, and, if $n$ is integral, $\epsilon_{1,0}=\zeta d$ for some scalar $\zeta$. Since $\epsilon_{1,0}$ does not enter the intertwining equation unless $n+j=1$ or $n+i=0$, the first sentence of Part (iii) now follows easily: the terms involving the cochains $\Delta_{n+j, n+i}$ cancel due to the conditions imposed by Part (i). For the second sentence of Part (iii), use Part (iii) of Theorem 7.10, the terms not involving $\partial_{u}$ cancel due to Part (i) of the current proposition.

Parts (iv) and (v) are similar, so we only discuss Part (iv). For $r \geq 3$, the $(r, 0)$-entry of the intertwining equation is

$$
\epsilon_{r} \bar{\pi}_{r, 0}^{\lambda, \mu}=\epsilon_{0} \bar{\pi}_{r, 0}^{\lambda^{\prime}, \mu^{\prime}}+\zeta \bar{\pi}_{r, 1}^{\lambda^{\prime}, \mu^{\prime}} d
$$

Again, the terms involving the cochains $\Delta_{n+j, n+i}$ cancel due to the conditions imposed by Parts (i) and (ii). To finish the proof, use Lemma 7.2 together with the fact that $d$ is an a-map to see that $\beta_{r, 1} \circ d=\beta_{r, 0}$.

Proofs of Propositions 4.1, 4.2, and 4.4 and Theorem 4.3. Just as the non-resonant equivalence results are all corollaries of Proposition 7.6, the resonant results are all corollaries of Proposition 7.14. We shall only give the details in the most important cases.

In Proposition 4.4, all of the entries of the intertwining equation involved fall under Part (i) of Proposition 7.14. Therefore the proof goes exactly as for Theorem 3.4 .

The proof of Theorem 4.3 is similar: only Parts (i) and (ii) of Proposition 7.14 are involved. In the event that none of the four functions in the theorem vanish, solving (11) for $(n+i, n+j)$ equal to $(1,-1),(2,-1)$, and $(1,0)$ determines the scalars $\epsilon_{-1}, \epsilon_{0}, \epsilon_{1}$, and $\epsilon_{2}$ up to a common multiplicative scalar. Then solving it at the last entry $(2,0)$ gives the invariant $R$. The scalar $\zeta$ has no effect.

The $n=-2$ case of Proposition 4.2 follows from the $n=0$ case by duality. The $n=0$ case involves Part (iv) of Proposition 17.14, but if $b_{3,1} \neq 0$ we can pick $\zeta$ to satisfy the relevant equation, imposing no new condition. When $b_{3,1}=0, \zeta$ has no effect and we get the additional condition

$$
\bar{b}_{3,0}(\gamma, \delta) \epsilon_{3}=\bar{b}_{3,0}\left(\gamma^{\prime}, \delta^{\prime}\right) \epsilon_{0} .
$$

Using Theorem 7.10, one obtains $\bar{b}_{3,0}=-\frac{1}{2 \sqrt{3}}(\delta)_{3} \gamma^{1 / 2}\left(\frac{5}{6} B_{3,1}+\delta-3\right)$. Proposition 4.2 now follows easily.

7.3. Lacunary cases. First note that the $\operatorname{Vect}(\mathbb{R})$-module $\Psi_{\lambda, \mu}^{k \text {,lac }}$ exists because the entries $\pi_{m+1, m}$ and $\bar{\pi}_{m+1, m}$ on the first subdiagonal are all zero except for $\bar{\pi}_{1,0}$. Each lacunary module still has either a projective quantization $\mathrm{PQ}_{\lambda, \mu}$ or an affine quantization $\overline{\mathrm{PQ}}_{\lambda, \mu}$, depending on whether or not it is resonant. To obtain the associated representation $\pi$ or $\bar{\pi}$, simply delete from the non-lacunary $\pi$ or $\bar{\pi}$ the rows and columns passing through the diagonal entries $\pi_{m, m}$ or $\bar{\pi}_{m, m}$ corresponding to the excised composition series modules $\mathcal{F}_{m}$.

We will only give the proofs of Theorems 5.3 and 5.5 , the other lacunary results are proven similarly. 
Proof of Theorem 5.3. In the non-resonant case we obtain the representations

$$
\pi^{\lambda, \mu}=\left(\begin{array}{ccc}
L_{n} & & \\
\pi_{n, \mu}^{\lambda, \mu, n} & L_{n+2} & \\
\pi_{n+4, n}^{\lambda, \mu} & \pi_{n+4, n+2}^{\lambda, \mu} & L_{n+4}
\end{array}\right), \quad \pi^{\lambda^{\prime}, \mu^{\prime}}=\left(\begin{array}{ccc}
L_{n} & & \\
\pi_{n+\mu^{\prime}}^{\lambda^{\prime}, \mu^{\prime}} & L_{n+2} & \\
\pi_{n+4, n}^{\lambda^{\prime}, \mu^{\prime}, n} & \pi_{n+4, n+2}^{\lambda^{\prime}, \mu^{\prime}} & L_{n+4}
\end{array}\right)
$$

both acting on $\mathcal{F}_{n} \oplus \mathcal{F}_{n+2} \oplus \mathcal{F}_{n+4}$. The subdiagonal entries of these matrices are still given by the formulas of Corollary 7.3 and Theorem 7.4 . The reasoning of Proposition 7.6 shows that any equivalence $\epsilon$ between them must be block-diagonal with scalar diagonal entries $\epsilon_{n}, \epsilon_{n+2}$, and $\epsilon_{n+4}$. If none of the subdiagonal entries is zero, then solving (11) at the entries $(n+2, n)$ and $(n+4, n+2)$ determines these three scalars up to a common multiplicative scalar, and solving it at $(n+4, n)$ gives the invariant $I_{n}$. This proves the theorem in the non-resonant cases. The only resonant cases involve only Part (i) of Theorem 7.14, so the non-resonant proof is valid also for them.

Proof of Theorem 5.5. Here the representation $\pi^{\lambda, \mu}$ is

$$
\left(\begin{array}{cccc}
L_{n} & & & \\
\pi_{n+2}^{\lambda, \mu} & L_{n+2} & & \\
\pi_{n+3}^{\lambda, \mu} & 0 & L_{n+3} & \\
\pi_{n+5, n}^{\lambda, \mu} & \pi_{n+5, n+2}^{\lambda, \mu} & \pi_{n+5, n+3}^{\lambda, \mu} & L_{n+5}
\end{array}\right) .
$$

If none of the subdiagonal entries is zero, then solving (11) at the entries $(n+2, n)$, $(n+3, n)$, and $(n+5, n+3)$ determines the scalars $\epsilon_{n}, \epsilon_{n+2}, \epsilon_{n+3}$, and $\epsilon_{n+5}$ up to a common multiplicative scalar, and solving it at $(n+5, n+2)$ gives the invariant $M_{n}$.

At this point a new phenomenon occurs. One would expect (11) to impose a new condition at $(n+5, n)$, but since $n$ is not allowed to be -4 or 0 , the following proposition shows that it does not: (11) is automatically satisfied at $(n+5, n)$ if it is satisfied at the other entries. Therefore the proof is complete.

Proposition 7.15. Fix complex scalars $n_{1}, \ldots, n_{l}$ such that $n_{i}-n_{i-1} \in \mathbb{Z}^{+}$for $1 \leq i \leq l$. Suppose that $\sigma$ and $\sigma^{\prime}$ are two representations of $\operatorname{Vect}(\mathbb{R})$ on $\bigoplus_{i=1}^{l} \mathcal{F}_{n_{i}}$. Regard them as block matrices with entries $\sigma_{i, j}: \operatorname{Vect}(\mathbb{R}) \rightarrow \operatorname{Hom}\left(\mathcal{F}_{n_{j}}, \mathcal{F}_{n_{i}}\right)$. Assume that $\sigma$ has the following form:

(i) $\sigma$ is lower triangular: $\sigma_{i, j}=0$ for $i<j$.

(ii) The diagonal entries are the Lie actions: $\sigma_{i, i}=L_{n_{i}}$.

(iii) For $n_{j+1}-n_{j}=1$ and $n_{j} \neq 0, \sigma_{j+1, j}=0$.

(iv) For $n_{i}-n_{j} \geq 2$ and $n_{i}+n_{j} \neq 1$, there is a scalar $c_{i, j}$ such that

$$
\sigma_{i, j}=c_{i, j} \beta_{n_{j}, n_{i}} .
$$

(v) For $i>j$ and $n_{i}+n_{j}=1$, there is a scalar $c_{i, j}$ such that

$$
\sigma_{i, j}=c_{i, j} \alpha_{n_{j}, n_{i}} \text {. }
$$

Assume that $\sigma^{\prime}$ has the same form with scalars $c_{i, j}^{\prime}$ replacing the $c_{i, j}$.

Suppose that $\epsilon$ is an endomorphism of $\bigoplus_{i=1}^{l} \mathcal{F}_{n_{i}}$ whose block matrix is diagonal, with non-zero scalars $\epsilon_{1}, \ldots, \epsilon_{l}$ on the diagonal satisfying

$$
c_{i, j} \epsilon_{i}=c_{i, j}^{\prime} \epsilon_{j}
$$


for all $(i, j)$ such that either $n_{i}-n_{j} \in\{2,3,4\}$ or $\left(n_{i}, n_{j}\right)$ is one of the following:

$$
(1,0), \quad(1,-4), \quad(5,0), \quad \frac{1}{2}(7 \pm \sqrt{19},-5 \pm \sqrt{19}) .
$$

Then $c_{i, j} \epsilon_{i}=c_{i, j}^{\prime} \epsilon_{j}$ for all $(i, j)$, and $\epsilon$ is an intertwining map from $\sigma$ to $\sigma^{\prime}$.

Proof. Replacing $\sigma$ with $\epsilon \circ \sigma \circ \epsilon^{-1}$, we reduce to proving that the entries $\sigma_{i, j}$ with $2 \leq n_{i}-n_{j} \leq 4$ or $\left(n_{i}, n_{j}\right)$ as in (12) determine $\sigma$. Recall the following elementary cohomological result: the condition that $\sigma$ be a representation is equivalent to the cup equation:

$$
0=\partial \sigma_{i, j}+\sum_{i>k>j} \sigma_{i, k} \cup \sigma_{k, j} \text { for all } i>j .
$$

Thus $c_{i, j}$ is determined by the entries on the higher subdiagonals whenever $\partial \beta_{n_{j}, n_{i}}$ (or $\partial \alpha_{n_{j}, n_{i}}$ if $n_{i}+n_{j}=1$ ) is non-zero. The result now follows from the wellknown theorem of Feigin and Fuchs on the cohomology of Vect $(\mathbb{R})$ FF80, of which a small part tells us that $\partial \beta_{\lambda, \mu}=0$ if and only if $\mu-\lambda \in\{2,3,4\}$ or $(\lambda, \mu)$ is one of $(-4,1),(0,5)$, or $\frac{1}{2}(-5 \pm \sqrt{19}, 7 \pm \sqrt{19})$, and $\partial \alpha_{\lambda, 1-\lambda}=0$ if and only if $\lambda \in\left\{0,-\frac{1}{2},-1,-\frac{3}{2}\right\}$.

\section{Open PROBLEMS}

8.1. The 1-dimensional case. Over $\mathbb{R}$, the main open problem is to answer the equivalence question in lengths $l>5$. In the resonant case one could try to do this with Proposition 7.14 and the formulas of Theorem 17.10, but we have not taken any steps in this direction. In the non-resonant case the necessary tools are Proposition 7.6 and the formula of Theorem [7.4. Consider the case of length 6 . The first point is that by Proposition 7.15, the $(n+5, n)$ entry of (11) does not impose any new condition on $\epsilon$. Therefore the method used to prove Theorem 3.4 yields the following proposition.

Proposition 8.1. For $n$ non-resonant, the subquotients $\mathrm{SQ}_{\lambda, \mu}^{\delta-n, 6}$ and $\mathrm{SQ}_{\lambda^{\prime}, \mu^{\prime}}^{\delta^{\prime}-n}$ are equivalent if and only if the two pairs of length 5 subquotients $\left(\mathrm{SQ}_{\lambda, \mu}^{\delta-n, 5}, \mathrm{SQ}_{\lambda^{\prime}, \mu^{\prime}}^{\delta^{\prime}-n}\right)$ and $\left(\mathrm{SQ}_{\lambda, \mu}^{\delta-n-1,5}, \mathrm{SQ}_{\lambda^{\prime}, \mu^{\prime}}^{\delta^{\prime}-n-1,5}\right)$ are both equivalent.

Thus in the generic case where both pairs of length 5 subquotients fall under Part (v) of Theorem 3.4 the four functions $I_{n}, J_{n}, I_{n+1}$, and $J_{n+1}$ are complete invariants for the length 6 equivalence classes $\operatorname{EC}_{n}^{6}(\gamma, \delta)$. Similarly, in the nonresonant length 7 case the entries at $(n+5, n)$ and $(n+6, n+1)$ do not impose new conditions, and the entry at $(n+6, n)$ can do so only when the Feigin-Fuchs cocycles are involved, at $2 n=-5 \pm \sqrt{19}$. It would be interesting if these special values of $n$ arise in the answer to the length 7 equivalence question, but we expect that they do not: we conjecture that in the non-resonant case, the only equivalences in lengths $l \geq 6$ are given by conjugation and the de Rham differential, as explained in Lemmas 2.2 and 2.5, respectively. Despite substantial effort and use of computers we have been unable to prove this conjecture in length 6 or 7 , but it is relatively easy to prove in length 8 and hence in all higher lengths. We simply state the result here; we plan to give the proof in a future article treating also the length 6 and 7 cases. 
Proposition 8.2. For $n$ non-resonant and $l \geq 8, \mathrm{SQ}_{\lambda, \mu}^{\delta-n, l}$ and $\mathrm{SQ}_{\lambda^{\prime}, \mu^{\prime}}^{\delta^{\prime}-n}$ are equivalent if and only if their parameters $(\gamma, \delta)$ and $\left(\gamma^{\prime}, \delta^{\prime}\right)$ are either equal, in which case the modules are either conjugate or themselves equal, or make up the pair $\left(3(\nu+1)^{2}, \nu\right),\left(3 \nu^{2}, \nu+1\right)$ for some $\nu$, the case of Lemma 2.5 .

We remark that analysis of the equivalence classes of the lacunary modules $\Psi_{\lambda, \mu}^{\delta-n \text {, lac }} / \Psi_{\lambda, \mu}^{\delta-n-5 \text {, lac }}$ may be seen as an interesting "warm-up" problem for the equivalence question in length 7 , as they are subquotients of $\mathrm{SQ}_{\lambda, \mu}^{\delta-n, 7}$. These modules have composition series $\left\{\mathcal{F}_{n}, \mathcal{F}_{n+2}, \mathcal{F}_{n+3}, \mathcal{F}_{n+4}, \mathcal{F}_{n+6}\right\}$, and by Proposition 7.15 there are three rational invariants for their equivalence classes: $I_{n}, I_{n+2}$, and

$$
B_{n+6, n+3} B_{n+3, n} / B_{n+6, n+4} B_{n+4, n+2} B_{n+2, n},
$$

whose level curves form a pencil of cubics. Coupled with the SVC these invariants are complete, except possibly at $2 n=-5 \pm \sqrt{19}$, where one may need also the invariant $B_{n+6, n} / B_{n+6, n+3} B_{n+3, n}$, whose level curves form another pencil of cubics.

8.2. The multidimensional case. As stated in the introduction, over $\mathbb{R}^{m}$ with $m>1$ the equivalence question for differential operators was resolved for $\mathcal{D}_{\lambda, \mu}^{k}$ in [DO97] and [Ma99], and for $\mathrm{SQ}_{\lambda, \lambda}^{k, l}$ in LO99. The more general case of $\mathrm{SQ}_{\lambda, \mu}^{k, l}$ has not yet been studied. In all these settings the results are less rich than for $m=1$ because the composition series determines not just $n=\delta-k$, but also $\delta$ and $k$ themselves.

It may be possible to recover the richness of the 1-dimensional case with some combination of the following natural generalizations. First, one can consider all intertwining maps between subquotients, as in Ma99, rather than only equivalences. Second, for $m>1$ tensor field modules are the correct generalization of tensor density modules. These are $\operatorname{Vect}\left(\mathbb{R}^{m}\right)$-modules co-induced from finite-dimensional irreducible representations of the Lie subalgebra $\operatorname{Vect}_{0}\left(\mathbb{R}^{m}\right)$ of vector fields vanishing at 0 . Thus the scalar parameter $\lambda$ in $\mathcal{F}_{\lambda}\left(\mathbb{R}^{m}\right)$ becomes the lowest weight of a finite-dimensional irreducible module of the copy of $\mathfrak{g l}_{m}$ in $\operatorname{Vect}\left(\mathbb{R}^{m}\right)$ spanned by $\left\{x_{i} \partial_{x_{j}}: 1 \leq i, j \leq m\right\}$ (see for example Co09]). One should consider subquotients of modules of differential operators between arbitrary tensor field modules. Finally, it may be appropriate to admit pseudodifferential operator modules, although this is complicated by the fact that their symbol modules are co-induced from infinite-dimensional modules of $\operatorname{Vect}_{0}\left(\mathbb{R}^{m}\right)$.

\section{REFERENCES}

[CMZ97] Paula Beazley Cohen, Yuri Manin, and Don Zagier, Automorphic pseudodifferential operators, Algebraic aspects of integrable systems, Progr. Nonlinear Differential Equations Appl., vol. 26, Birkhäuser Boston, Boston, MA, 1997, pp. 17-47. MR.1418868 (98e:11054)

[Co05] Charles H. Conley, Bounded subquotients of pseudodifferential operator modules, Comm. Math. Phys. 257 (2005), no. 3, 641-657, DOI 10.1007/s00220-005-1327-4. MR2164946 (2006f:17028)

[Co09] Charles H. Conley, Quantizations of modules of differential operators, Symmetry in mathematics and physics, Contemp. Math., vol. 490, Amer. Math. Soc., Providence, RI, 2009, pp. 61-81, DOI 10.1090/conm/490/09587. MR2555970(2010k:17034)

[CS04] Charles H. Conley and Mark R. Sepanski, Singular projective bases and the affine Bol operator, Adv. in Appl. Math. 33 (2004), no. 1, 158-191, DOI 10.1016/j.aam.2003.08.004. MR2064602(2005c:17032) 
[DO97] C. Duval and V. Yu. Ovsienko, Space of second-order linear differential operators as a module over the Lie algebra of vector fields, Adv. Math. 132 (1997), no. 2, 316-333, DOI 10.1006/aima.1997.1683. MR 1491445 (99b:17028)

[FF80] B. L. Fergin and D. B. Fuks, Homology of the Lie algebra of vector fields on the line (Russian), Funktsional. Anal. i Prilozhen. 14 (1980), no. 3, 45-60, 96. MR583800 (82b:17017)

[Ga00] H. Gargoubi, Sur la géométrie de l'espace des opérateurs différentiels linéaires sur $\mathbf{R}$ (French, with English and French summaries), Bull. Soc. Roy. Sci. Liège 69 (2000), no. 1, 21-47. MR1766660 (2001f:17044)

[GO96] H. Gargoubi and V. Yu. Ovsienko, Space of linear differential operators on the real line as a module over the Lie algebra of vector fields, Internat. Math. Res. Notices 5 (1996), 235-251, DOI 10.1155/S1073792896000177. MR1383757 (96m:17041)

[La12] Jeannette M. Larsen, Equivalence classes of subquotients of pseudodifferential operator modules on the line, ProQuest LLC, Ann Arbor, MI. Thesis (Ph.D.)-University of North Texas, 2012. MR3152666

[LMT96] P. B. A. Lecomte, P. Mathonet, and E. Tousset, Comparison of some modules of the Lie algebra of vector fields, Indag. Math. (N.S.) 7 (1996), no. 4, 461-471, DOI 10.1016/S0019-3577(97)89133-1. MR1620108(99c:17038)

[LO99] P. B. A. Lecomte and V. Yu. Ovsienko, Projectively equivariant symbol calculus, Lett. Math. Phys. 49 (1999), no. 3, 173-196, DOI 10.1023/A:1007662702470. MR.1743456 (2001e:58035)

[Ma99] P. Mathonet, Intertwining operators between some spaces of differential operators on a manifold, Comm. Algebra 27 (1999), no. 2, 755-776, DOI 10.1080/00927879908826459. MR 1671975 (99m:58209)

[Ma00] P. Mathonet, Geometric quantities associated to differential operators, Comm. Algebra 28 (2000), no. 2, 699-718, DOI 10.1080/00927870008826853. MR.1736756|(2001a:17036)

Department of Mathematics, University of North Texas, Denton, Texas 76203

E-mail address: conley@unt.edu

Department of Mathematics, University of Texas at Tyler, Tyler, Texas 75799

E-mail address: jlarsen@uttyler.edu 